\title{
Multivariate patterns of brain-behavior associations across the adult lifespan
}

\author{
Gaelle E. Doucet ${ }^{1,4}$, Noah Hamlin ${ }^{1}$, Anna West ${ }^{1}$, Jordanna A. Kruse ${ }^{1}$, Dominik A. Moser ${ }^{2,3}$, Tony \\ W. Wilson ${ }^{1,4}$ \\ ${ }^{1}$ Institute for Human Neuroscience, Boys Town National Research Hospital, Omaha, NE 68010, USA \\ ${ }^{2}$ Institute of Psychology, University of Bern, Bern, Switzerland \\ ${ }^{3}$ Child and Adolescent Psychiatry, University Hospital Lausanne, Lausanne, Switzerland \\ ${ }^{4}$ Department of Pharmacology and Neuroscience, Creighton University School of Medicine, Omaha, NE 68178, USA
}

Correspondence to: Gaelle E. Doucet; email: gaelle.doucet@boystown.org

Keywords: healthy aging, higher-order cognition, multivariate analyses, MRI, brain networks

Received: October 28, $2021 \quad$ Accepted: December 20, $2021 \quad$ Published: January 10, 2022

Copyright: (c) 2022 Doucet et al. This is an open access article distributed under the terms of the Creative Commons Attribution License (CC BY 3.0), which permits unrestricted use, distribution, and reproduction in any medium, provided the original author and source are credited.

\section{ABSTRACT}

The nature of brain-behavior covariations with increasing age is poorly understood. In the current study, we used a multivariate approach to investigate the covariation between behavioral-health variables and brain features across adulthood. We recruited healthy adults aged 20-73 years-old (29 younger, mean age $=25.6$ years; 30 older, mean age $=\mathbf{6 2 . 5}$ years), and collected structural and functional MRI (s/fMRI) during a restingstate and three tasks. From the sMRI, we extracted cortical thickness and subcortical volumes; from the fMRI, we extracted activation peaks and functional network connectivity (FNC) for each task. We conducted canonical correlation analyses between behavioral-health variables and the sMRI, or the fMRI variables, across all participants. We found significant covariations for both types of neuroimaging phenotypes $(p s=0.0004)$ across all individuals, with cognitive capacity and age being the largest opposite contributors. We further identified different variables contributing to the models across phenotypes and age groups. Particularly, we found behavior was associated with different neuroimaging patterns between the younger and older groups. Higher cognitive capacity was supported by activation and FNC within the executive networks in the younger adults, while it was supported by the visual networks' FNC in the older adults. This study highlights how the brainbehavior covariations vary across adulthood and provides further support that cognitive performance relies on regional recruitment that differs between older and younger individuals.

\section{INTRODUCTION}

Brain organization throughout the lifespan has been associated with multiple genetic, molecular, behavioral, and environmental factors [1-6]. Large-scale studies have begun to address the complexity of the brain-behaviorenvironment associations. In the Human Connectome Project -Young Adult (HCP-YA), a U.S. study done on healthy adults aged 22-37 years, personal attributes and environmental factors were correlated with brain structure and functional connectivity following a positive-negative axis $[7,8]$. In other words, the majority of behavioral variables that correlated positively with more efficient brain patterns (e.g., stronger network integration) were positive subject traits and measures (such as education, income, IQ, life-satisfaction), while those that correlated negatively were mostly negative subject measures (such as body-mass index (BMI), smoking, age). This finding has been consistently reproduced using independent samples of young adults [9], youth [10-12] and clinical samples [9, 13-16].

Throughout adulthood, age has been robustly established as one of the strongest negative contributors to brain 
function using multivariate $[7,8]$ and univariate [17-20] approaches. Healthy aging has been associated with regional brain atrophy $[17,21,22]$, reduced brain network integrity [20, 23-25] and changes in regional connectivity [20, 24-26]. Beyond this negative impact, a study by Miller et al. (2016) [17] that involved 5,000 participants aged 44-78 years old suggested that aging alters the brainbehavior-environment associations in two ways. First, these associations may vary by age range (i.e., early versus middle versus late adulthood). Second, within older adults, age-related cognitive decline seems to be a major source of variability for these associations, suggesting that agerelated impact varies by neural parameter and cognitive function investigated [17]. However, there is a limited number of neuroimaging studies that focused on multivariate associations in late adulthood or across the adult lifespan, even though a key goal of cognitive neuroscience and multiple federal funding initiatives is to characterize how age-related variation in brain organization relates to cognitive, psychosocial and environmental factors, as this may help identify the relevant brain mechanisms that signify the transition from healthy aging to neurodegenerative states [27, 28].

In this context, the aim of the present study was to further characterize the impact of healthy aging on patterns of multivariate covariation between nonimaging and imaging variables and identify the most salient features that drive these associations in older relative to younger healthy adults. To achieve this aim, we recruited 59 healthy adults (29 adults aged 19-32 years-old and 30 adults aged 55-72 years-old). We collected a high-resolution structural scan, three cognitive functional MRI (fMRI) tasks that related to working memory, visual episodic memory and language function, respectively, and a resting-state fMRI scan in one session. These tasks assess three of the most critical components of cognitive processing, underlying many more complex functions. Using the structural scan, we extracted structural morphometric measures (subcortical volumes and cortical thickness), while with the fMRI data we extracted individual measures of task-related and resting-state functional network connectivity (FNC) and task-related brain activation. We extracted both types of brain functional features because they: (a) are among the most commonly analyzed measures in fMRI, (b) have been suggested to reflect different brain mechanisms $[29,30]$ and (c) are related to distinct behavioral and cognitive features [9, 17]. Lastly, in aging models, it appears that functional connectivity versus task-based activation techniques may reveal different aspects of recruitment of additional regions to support and/or compensate cognitive processing [3134]. Overall, we aimed to: (1) test the relative sensitivity of these brain features (structural and functional) to maintain optimal cognitive function and health, and (2) compare these associations between early and late adulthood. In this context, we ascertained non-imaging/health factors that related to medical history, metabolism, cognition, lifestyle factors and physical health ( $n=59$ variables). These variables were selected based on previous fMRI studies which demonstrated their relevance in understanding the covariation linking brain activity, demographics, and behavior [7, 8, 17]. Using these multimodal data, we conducted sparse canonical correlation analyses (sCCA) $[35,36]$ to investigate the underlying relationship between the neuroimaging and health/demographic/lifestyle factors in the whole sample, and further to identify the differential weights of contribution of the top variables in each model in the younger versus older adults. We used sCCA because this multivariate method is ideally suited for predicting one dataset from the other while accounting for correlations between variables [36, 37]. CCA is a powerful multivariate tool to simultaneously investigate relationships among multiple variables and/or datasets, and further enables one to separate distinct biological processes with opposing relationships between variables $[17,36]$. In particular, we chose to conduct sCCA because it is tailored to the analyses of highdimensional datasets in which variables are expected to be correlated and does not require data reduction. It has the further advantage over classic CCA that more observations than participants is acceptable and produces reliable results even in smaller samples [36, 38]. Considering preexisting evidence, our working hypotheses were that beyond an overall negative effect of age at the level of the whole sample, the brainbehavior covariation would vary between the two age groups. Particularly, we expected that: (i) age would have a stronger (negative) impact on brain efficiency in the older group, and (ii) top contributors to the brainbehavior covariations would differ between the two groups. In particular, we expected that health- and metabolism-related measures (e.g., glucose rate, physical activity) would contribute more to brain integrity in older than in younger adults. We also hypothesized that cognitive variables shown as strong positive predictors of brain integrity in youth and young adults $[7-9,11]$ would show a lower contribution in older adults, because healthy aging has been typically associated with slight declines and slowing in general cognitive function [39, 40]. Finally, (iii) older adults would show a more widespread functional and structural regional contribution to support higher-order cognitive function when compared to the younger adults. The latter follows the compensation-related utilization of neural circuits hypothesis (CRUNCH) [40], and more recently the revised scaffolding theory of aging and cognition (STAC-r) model [34, 41]. These aging models support the overall idea that age-specific 
increases in regional recruitment provide support to maintain higher cognitive performance in older individuals, with this positive association enhanced by enriched lifestyle (e.g., higher physical activity). Lastly, we hypothesized that measures of structural morphometry would emerge as stronger contributors to behavior than the functional features as our previous study found in young adults [9].

\section{RESULTS}

\section{Sparse CCA between non-imaging and sMRI datasets}

Across all participants, we found a significant association between the non-imaging dataset and the sMRI dataset $(\mathrm{r}=0.785, p=0.0004$; Figure $1 \mathrm{~A})$. In the non-imaging dataset, the highest positive weights were attributed to measures related to intelligence and higher cognitive function (verbal fluency, block design), better accuracy during the fMRI tasks, and emotions related to negative affect and perceived negative social relationships (Figure 1B, Supplementary Table 1). In contrast, older age, emotions related to well-being, and lower motor dexterity (e.g., slower reaction times) displayed the strongest negative contribution. In the imaging dataset, the structural features with the highest positive contributions were the cortical thickness of lateral and medial frontal regions as well as superior temporal cortex (Figure 1C, Supplementary Table 2). In contrast, the top negative structural contributors were the volume of the lateral ventricles.

We further identified the top contributors to the covariation within each age group (Figure 2). While we only report and discuss the top variables, all variable contributions are provided in Supplementary Tables 1 and 2. In the non-imaging dataset, the variables with the highest correlations with the sMRI dataset were mostly different between the two groups. In the younger group, the non-imaging variables with the strongest contributions were related to higher BMI, reaction times, apathy and cognitive ability. In the imaging dataset, cortical thickness measures most highly correlated with the non-imaging variate were widely distributed in the parietal, temporal, frontal and visual cortex. Particularly, cortical thickness of the primary cortices showed a negative association with the behavioral variables while cortical thickness of associative cortices were positively associated

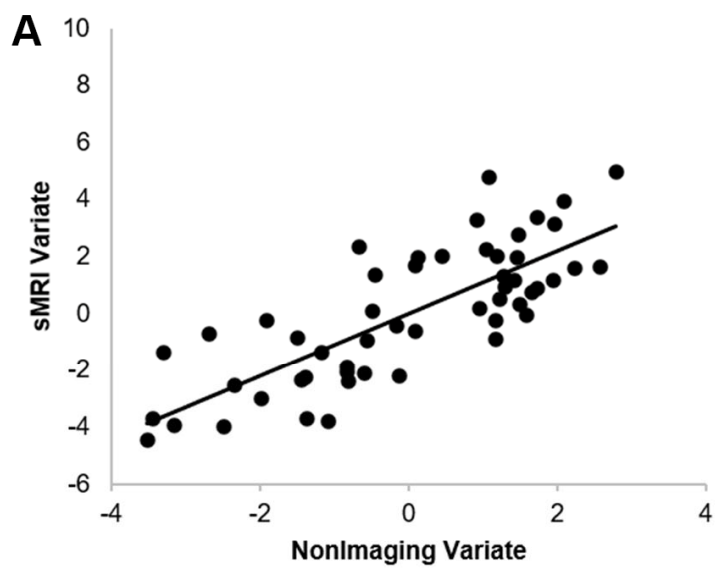

C
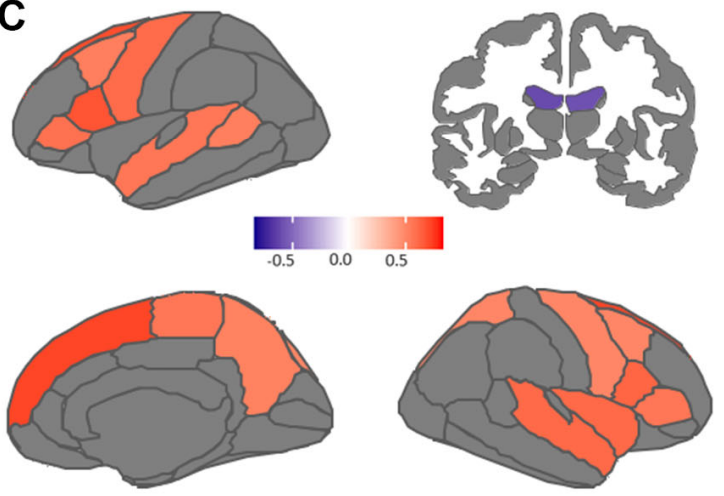

B

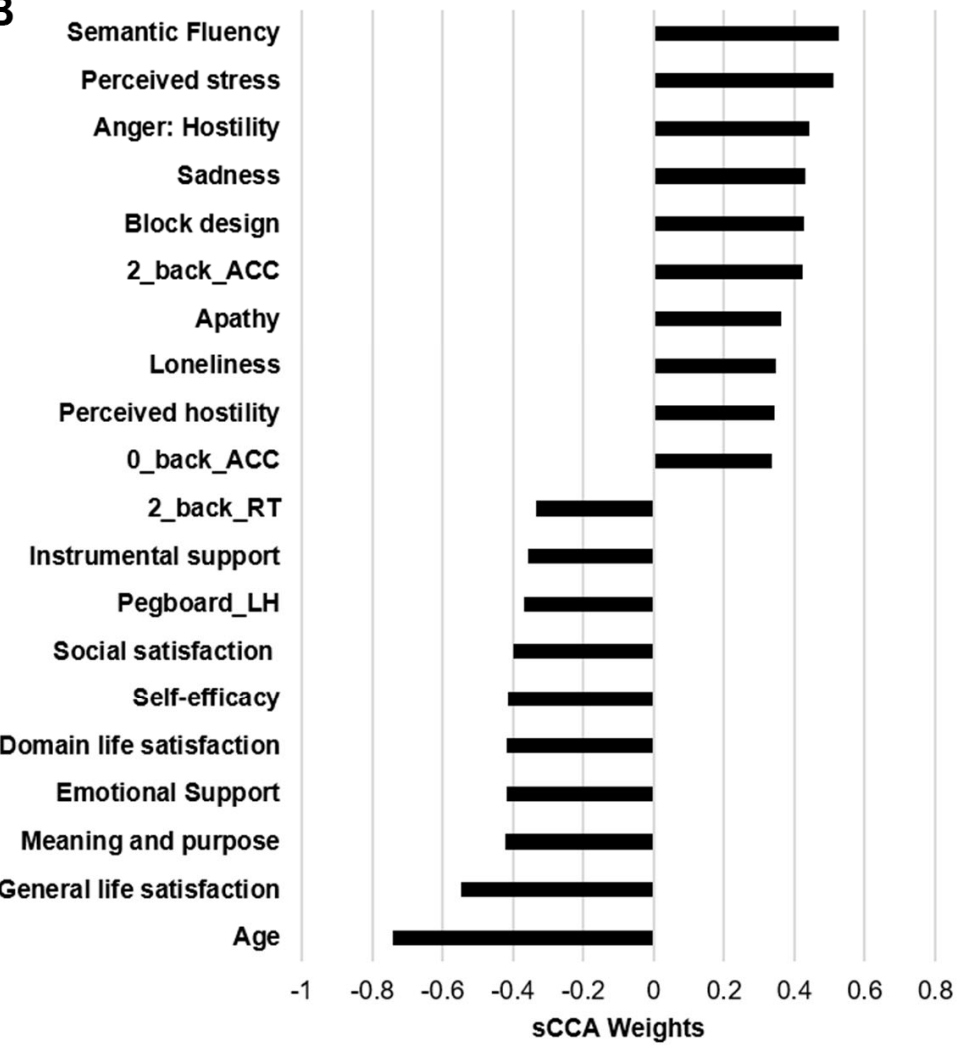

Figure 1. Results of the SCCA between non-imaging and SMRI datasets across all participants. (A) Significant correlation across all participants $(r=0.612, p=0.0001)$. (B) Top behavioral-health variables most strongly associated with the imaging variate. (C) Top sMRI variables positively associated with the behavioral-health variate. Details of each variable in Supplementary Table 5 . Contributions of all variables are provided in Supplementary Tables 1 and 2. 
(Figure 2, Supplementary Table 2). In the older group, higher age, blood pressure as well as better life satisfaction were the variables with the strongest negative associations to the sMRI dataset, while better cognitive functions remained among the most positive variables. In the sMRI dataset, lateral ventricles and orbitofrontal regions were identified as variables with the most negative associations to the behavioral variables, in contrast cortical thickness of the dorsomedial frontal regions, pre- and postcentral gyri showed the strongest positive associations.

\section{Sparse CCA between non-imaging and fMRI datasets}

Across all participants, we found the sCCA significant between the non-imaging dataset and the fMRI variables $(\mathrm{r}=0.91, p=0.0004$; Figure 3; Supplementary Table 3). In the non-imaging dataset, the highest positive weights were attributed to better cognitive ability (such as matrix reasoning and block design, higher accuracy during the fMRI tasks), and stronger report of emotions related to negative affect. In contrast, the highest negative weights

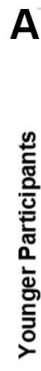

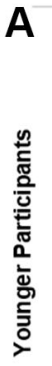
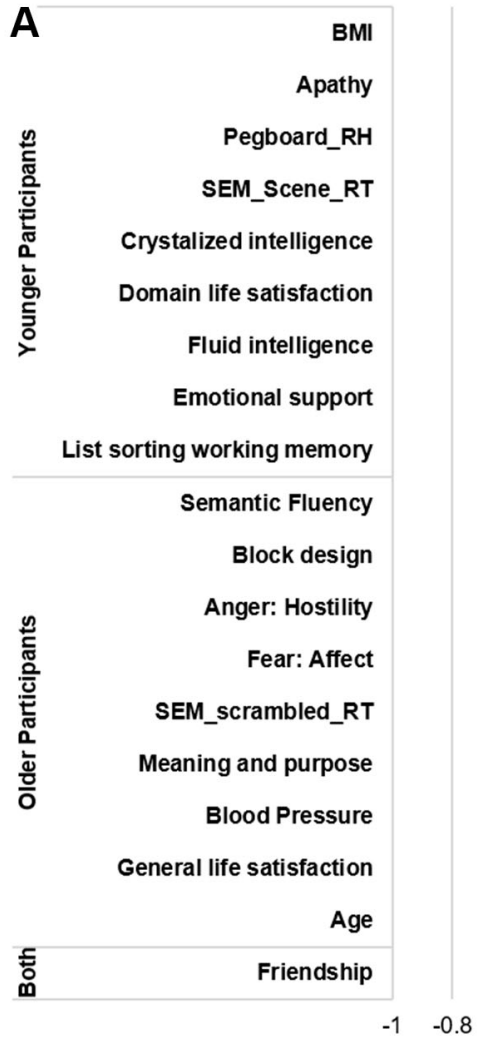

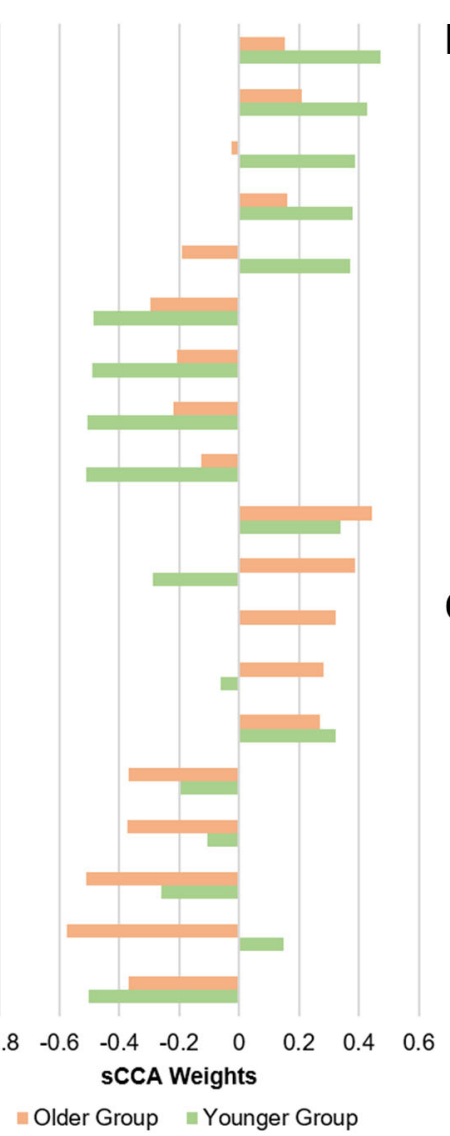

were largely attributed to older age, lower motor dexterity (higher time to complete a pegboard with either right or left hand), psychological well-being, and higher estimated average glucose (EAG) rate (Figure 3, Supplementary Table 3). In the imaging dataset, the FNC measures mostly showed a negative association while the activation variables were positive contributors. Both the FNC and activation measures most highly correlated with the non-imaging dataset were collected during the n-back and Scene Encoding Memory (SEM) tasks. The networks most involved were largely related to executive function (ECN/SAL) and primary cortices (SMN/VIS).

We further identified the top contributors to the covariation between fMRI and behavior features within each age group (Figure 4, Supplementary Table 3). In the non-imaging dataset, the variables with the highest correlations with the fMRI dataset were different between the two groups. In the younger group, the top contributors were relatively similar to the results of the overall sCCA. The non-imaging variables with the strongest negative contributions were related to older

\section{B Younger Participants}

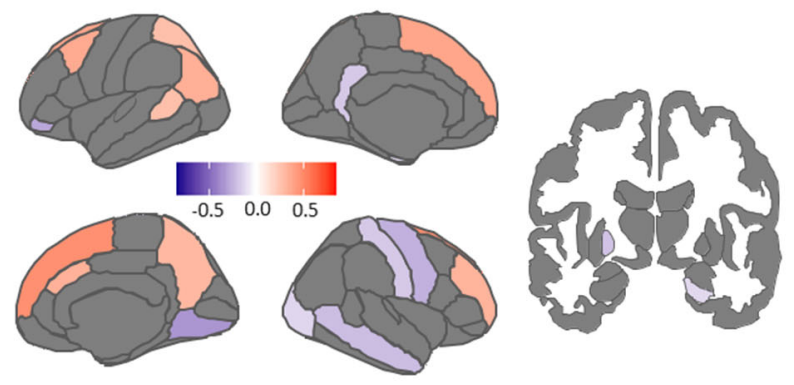

\section{Older Participants}

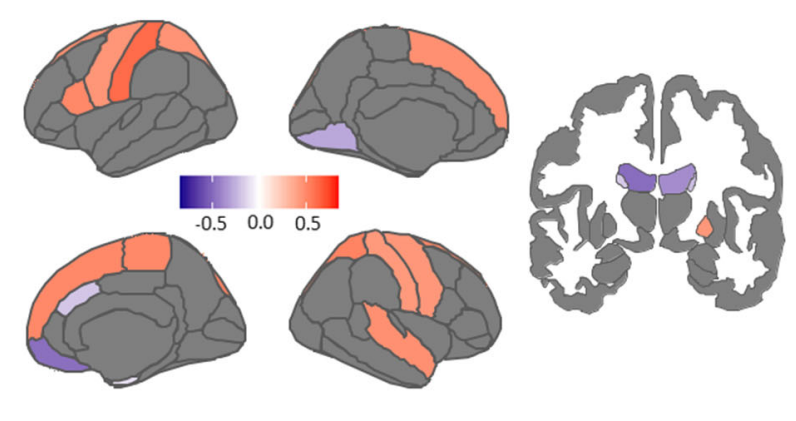

Figure 2. Top contributors in the sMRI sCCA in younger and older groups, separately. (A) Top behavioral-health variables most strongly associated with the imaging variate in each subgroup. (B) Top sMRI variables associated with the behavioral-health variate in the younger group. (C) Top sMRI variables associated with the behavioral-health variate in the older group. Details in Supplementary Tables 1 , 2 and 5 . 
age, motor dexterity, sleep quality during the weekend and positive affect; in contrast, estradiol rate, reading capacity and negative affect were positively associated with the fMRI dataset. In the imaging dataset, the FNC measures mostly showed a negative association while the activation variables were positive contributors. The FNC most highly correlated with the non-imaging dataset were collected during the Verb Generation (VG) and SEM tasks, largely involving networks related to executive function (ECN/SAL), primary cortices (SMN/VIS) and the default

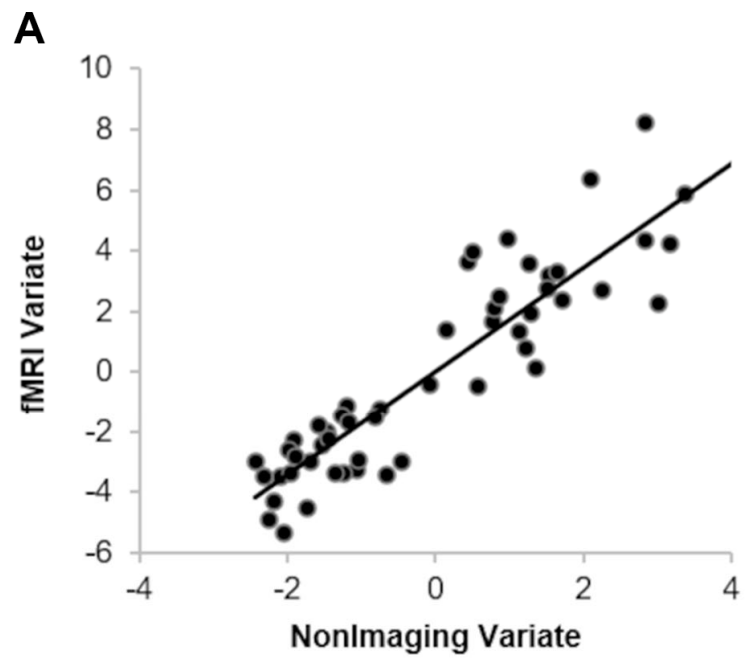

C

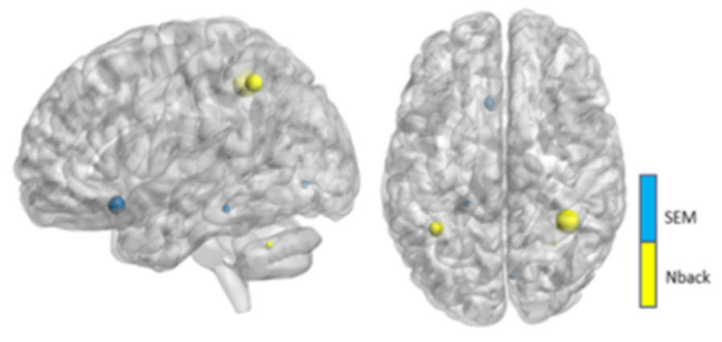

\section{B}

2_back_ACC
Perceived stress
Matrix reasoning
Block design
Anger: Hostility
Loneliness

Perceived hostility

Sadness

Perceived rejection

Fear: Somatic arousal

EAG

Self-efficacy

Instrumental support

Social satisfaction

Meaning and purpose

0_Back_MeanRT

General life satisfaction

Pegboard_LH

Pegboard_RH

Age
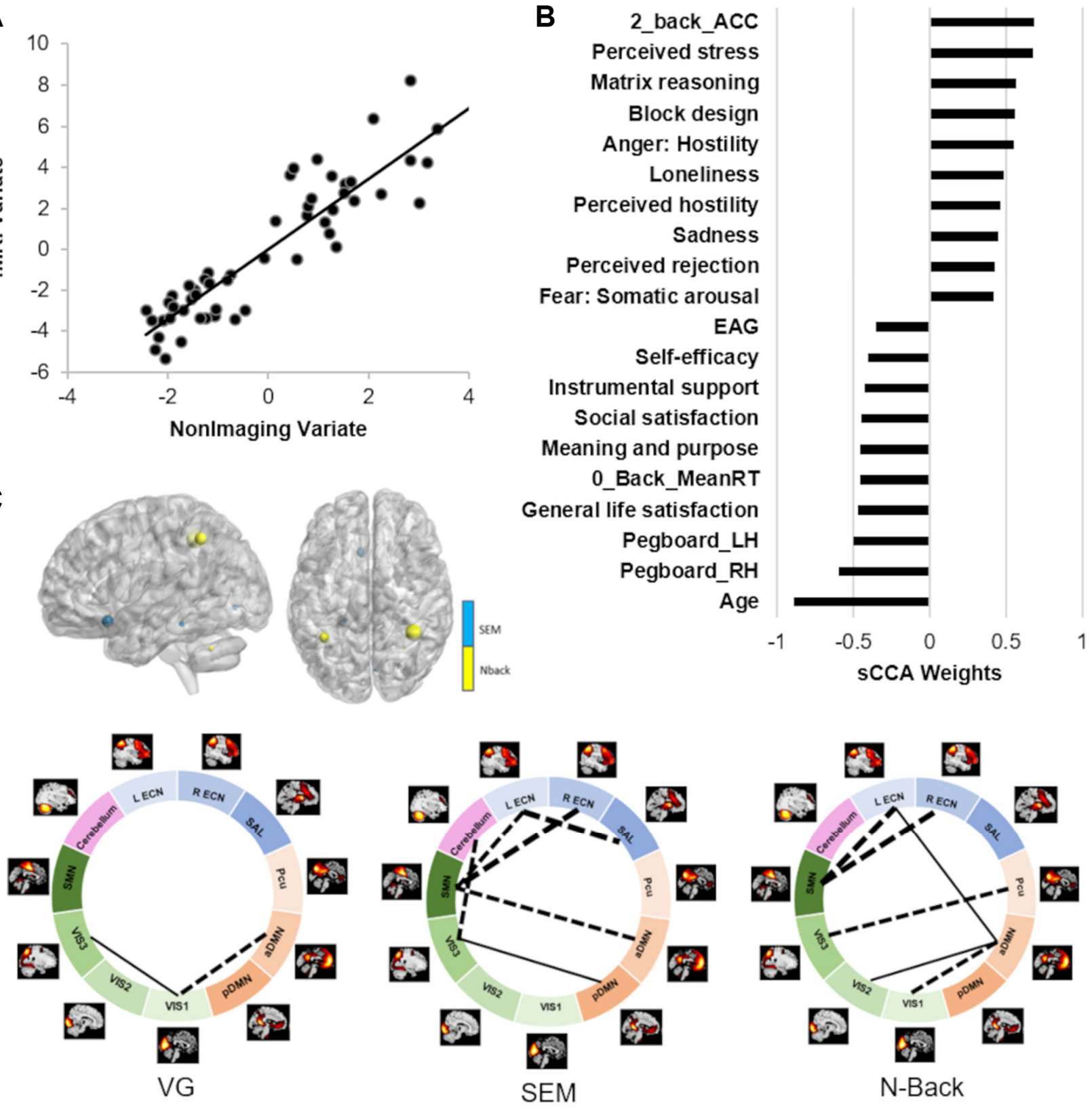

Figure 3. Results of the SCCA between non-imaging and fMRI datasets across all participants. (A) Significant correlation across all participants $(r=0.91, p=0.0004)$. (B) Top behavioral-health variables most strongly associated with the imaging variate. (C) Top fMRI features most strongly associated with the non-imaging variate. Dashed lines between networks indicate negative contributions of the FNC; solid lines between networks indicate positive contribution of the FNC. Details in Supplementary Tables 3 and 5. 
executive (ECNs) and default mode networks to a lower level.

\section{Reliability analyses}

To assess whether our overall results were robust, we 1) performed leave-one out (LOO) analysis for every participant; 2) computed a redundancy-reliability score
(Moser's RR-score) for each overall sample sCCA [42]. The RR-score is a measure of the stability of the variable-to-variate correlations and indicates whether results can be expected to be reliable independent of sample composition (see detail in the methods). The LOO analyses indicated that both overall sCCAs were very stable and did not show outliers (the weights of each LOO analysis correlated above 0.95 with the

\section{A

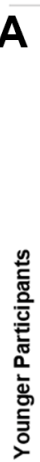

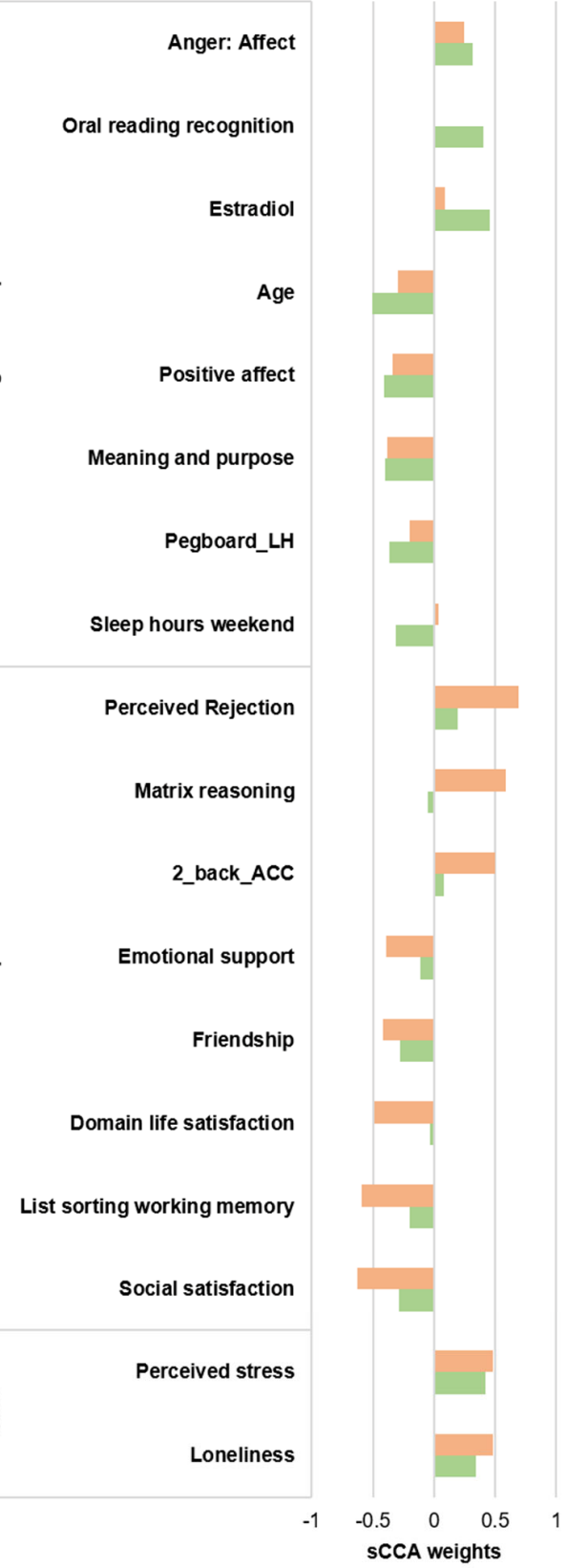

- Older Participants $\quad$ "Younger Participants

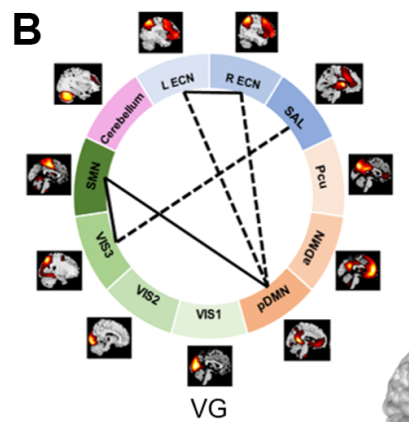

B

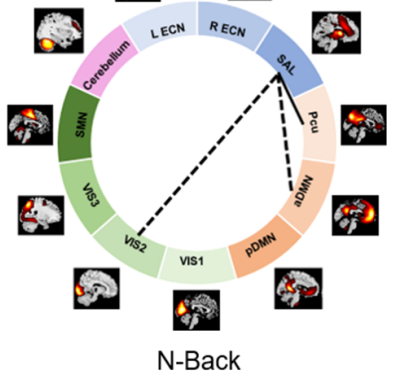

C

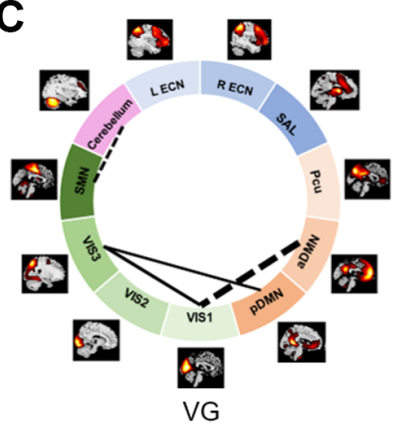

(1) B

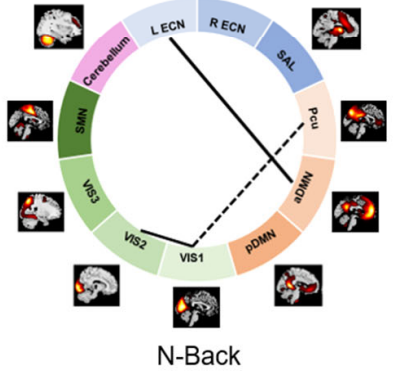

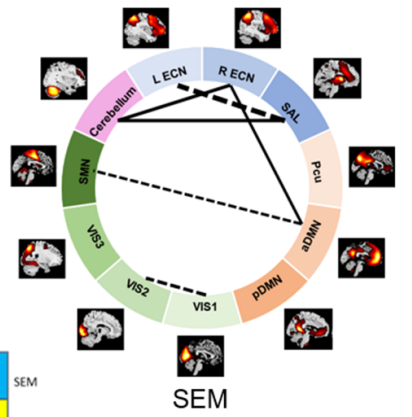

B
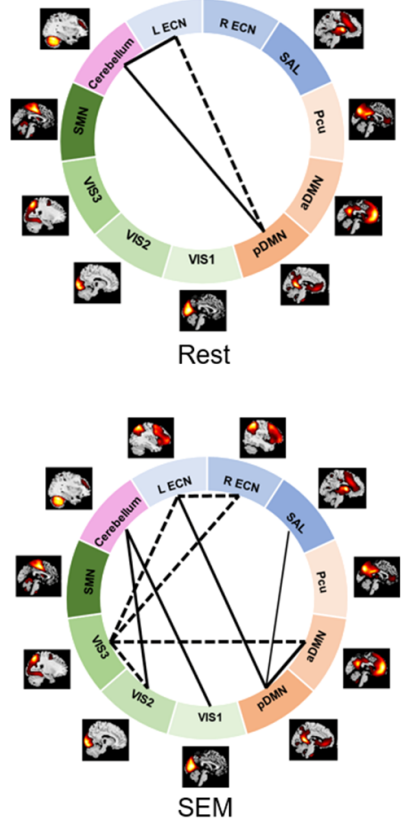

D

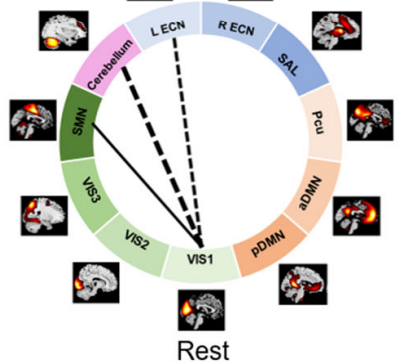

Figure 4. Top 10 features contributing to the fMRI SCCA in younger and older groups, separately. (A) Top behavioral-health variables most strongly associated with the imaging variate in each subgroup. (B) Top fMRI variables most strongly associated with the behavioral-health variate in the younger group. (C) Top fMRI variables most strongly associated with the behavioral-health variate in the older group. Dashed lines between networks indicate negative contributions of the FNC; solid lines between networks indicate positive contribution of the FNC. Details in Supplementary Tables 3 and 5. 
sCCA weights in the full dataset). The RR-scores indicated that both overall sCCAs yielded reliable solutions (sMRI: median RR-score was 0.88 , SD = 0.05; fMRI: median 0.87 , SD $=0.11$, Supplementary Figure 1). Lastly, when half the sample was randomly resampled 5000 times, both overall sCCAs yielded similar correlations. We then applied the weights of each sCCA resample to the remaining half of the sample (using it as a test-set). The mean (standarddeviation) of the scores indicated that the sCCA results held predictive value for both sCCAs (sMRI: $\mathrm{r}=0.55$ (0.11); fMRI: $r=0.67(0.20)$ ).

\section{DISCUSSION}

The current study investigated the covariation between behavioral-health measures and brain structure and function across adulthood. While several previous studies have explored this relationship in adults, they typically focused on a limited age range, typically under 35 years (20 37 years for [7-9], 44-78 years for [17]). To our knowledge, the current study is among the first to investigate the multivariate associations among behavior and multimodal imaging phenotypes (structural MRI, task-based and resting-state fMRI) in a population that stretches across most of the adult lifespan (i.e., over 50 years; 20 to 73 years old). In line with our hypotheses, we found age-specific effects on brain-behavior covariations in healthy individuals. The impact of age was examined in two different and complementary methods: first, by examining the contribution of age in the brain-behavior associations across adulthood, and second by identifying the top variables that contributed to the covariations within older and younger age groups separately. Our results replicate previous findings focused on early adulthood, with the non-affective cognitive measures (e.g., IQ and other measures reflecting higher order cognitive functions) being the strongest positive contributions, and age and poorer motor dexterity being the most negative contributors to neuroimaging phenotypes [8, 9]. Beyond this general negative age-related impact, our findings revealed that such associations strongly vary by age group and neuroimaging phenotypes.

Across all participants, we found significant robust covariations between behavior and health measures and both brain structural morphometry and functional activation and connectivity measures. Better cognitive capacity typically had the strongest positive contribution to both brain structural and functional measures. In contrast, older age and slower motor dexterity showed a negative contribution. At the structural level, better cognition and younger age were related to thicker frontal and parietal cortices, among other widespread regions and larger subcortical volumes; while at the functional level, they were more strongly associated with greater activation and lower functional connectivity emerging from the brain networks supporting higher order cognitive functions, and particularly working memory (ECN, SAL). The degree of activation and functional connectivity of the working memory networks identified during the n-back task were among the strongest contributors to the nonimaging variables, which is consistent with both our previous findings $[7,9]$ and the fundamental role of these executive networks in everyday activity [43]. Interestingly, FNC and activations showed relatively opposite associations with behavior, where better cognition and younger age were associated with stronger activation and lower functional connectivity. In other words, older age was related to weaker activation in the memory networks and higher between-network functional connectivity. This finding supports the theory that healthy aging is associated with a progressive loss of functional integration and segregation of brain networks [20, 24, 44]. This opposition between activation and functional connectivity also points to the idea that these brain functional features are supported by different biological mechanisms [29].

In the fMRI sCCA, we found that the FNC features' contributions to the covariation varied by cognitive states. In other words, the FNC during n-back, SEM, $\mathrm{VG}$ and rest did not contribute equally to the behavioral variables. Recent fMRI studies have demonstrated that the functional network organization of the brain is not stationary and rather reconfigures dynamically as a function of the cognitive state $[45,46]$, predicts general cognitive abilities [47], and varies by cognitive demands during the task [48]. Our results are in line with these findings and the recent study by Varangis et al. [48] which further described that age, task domain and performance on the in-scanner task impact between-network connectivity. Further, in the current study, the strongest FNC contributors to the behavioral variate were largely between brain networks supporting lower order function (i.e., networks related to sensory and motor processing such as SMN and VIS) versus those related to higher order functions (i.e., ECN, SAL and DMN). Variability in such extrinsic-intrinsic network interactions have been linked to higher order cognitive capacity [49-52], which is again supported by the current findings.

Surprisingly, we also found a relatively strong association of affective cognitive measures with both brain structural and functional features, where higher reported positive affect and life satisfaction was negatively associated with brain measures and negative affect and higher stress were rather positively associated. The exact meaning of such finding is 
unclear. While we used the t-scores for each emotional measure which are age- and sex-corrected, we cannot exclude the possibility that age ultimately influenced the measure. Affective cognition has been shown to change throughout the lifespan, with older adults reporting improvement in emotional experience and an increased frequency of positive feelings, relative to younger adults [53], which is also what we report (see Supplementary Table 4). Other neuroimaging studies have independently reported association between affective cognition and working memory network activation [7], functional network connectivity [8, 13, $54,55]$ or structural morphometry [9]. It is also known from psychiatric studies that the activation of the working memory network is modulated by the level of depression and anxiety [56]. Overall, while the association between emotional well-being and brain functional and structural organization remains unclear and needs further investigation, we believe it likely reflects the complexity of the brain mechanisms behind emotional well-being and affective disorders across the lifespan $[13,57,58]$.

When investigating each age group separately, we found that the strongest contribution of the behavioral variables to the brain features typically differed between the younger and older adults, which is in line with our hypotheses. Our findings support that such differences between early and late adulthood are likely related to the non-linear impact of age on brain structure $[21,22]$, function $[24,33]$ and cognition $[39$, 40]. First, in the current study, age was identified as a strong negative contributor to brain structural morphometry in the older but not in the younger adults, and vice-versa in the fMRI sCCA. This is consistent with the recent studies done by the ENIGMA-Lifespan working group which have demonstrated an almost null impact of age on structural brain morphometry during early and middle adulthood, before showing a strong negative effect in late adulthood [21, 22]. Second, in the sMRI sCCA, we found a relatively unexpected association in young adults where BMI and motor dexterity were among the top positive contributors to variation in brain structural morphometry. However, it is important to note that at the brain level, cortical thickness showed a heterogeneous pattern with both thinning of the primary cortices and thickening of associative cortices in association with these behavioral variables. Higher BMI has been previously associated with reduced functional integration of both visual and sensorimotor networks in young adults [59]. In this context, the current study suggests that such impact may be directly linked to underlying structural thinning of these primary regions that respond to perception of food images and tastes [60]. Third, in the fMRI sCCA, the major differences in contributions between the two age groups were identified at the neuroimaging level. While the major functional features associated with the behavioral dataset in the younger adults largely followed those from the overall analysis (i.e., large involvement of the executive networks to support higher cognitive capacity), the older adults showed a different pattern where behavior was rather supported by changes in functional connectivity emerging from the visual networks. These findings are in line with aging models $[34,40]$ that support the idea of an agespecific regional recruitment of primary cortices to support and maintain relatively preserved performance in older individuals. The STAC-r model supports the idea that preserved cognition in older adults is partially supported by good brain efficiency, which involves compensatory mechanisms through the mediation of primary networks, despite being less efficient than in their youthful state $[32,34]$. With aging, this transition from executive to visual networks to support healthy behavior may reflect changes in biological mechanisms involving brain networks less impacted by aging [23, 34]. In fact, the overall negative impact of age on the functional integrity of the executive networks have been consistently reported [20, 23, 24, 44, 61], while the visual network is among the least impacted [23]. This relatively low impact of aging on the visual networks is likely related to their high structuralfunctional coherence [62], low inter- and intraindividual variability in functional activation [63], anatomical morphology [64, 65] and resting-state functional connectivity [66-68]. Together, the current findings indicate that, across adulthood: (a) there may be a progressive change in the functional support of healthy behavior and cognitive aging by recruiting more preserved networks such as the primary networks over executive control networks, as the latter become less efficient in late adulthood [44]; and (b) functional connectivity features may be more effective to highlight neural changes related to aging, than brain activation.

We found that variation in estradiol level was among the strongest variables associated with the fMRI dataset in the younger but not the older adults. We believe that this specific finding is related to a lack of variability in this hormone level in the older adults, as the majority of the older women reported being in menopause. However, this finding also underscores the impact of sexual hormones in brain functional organization in early adulthood.

Lastly, we expected a stronger contribution of healthrelated measures (such as physical activity, or EAG) to brain integrity in older adults but we did not reveal such effect. On the contrary, we found that BMI was a stronger contributor to brain structure in the younger 
group. With regard to physical activity, it is possible that only structured physical interventions have a significant impact on brain integrity and cognitive function [34], however, research studies on this topic remain relatively limited. While higher EAG contributed negatively to brain functional integrity across subjects, we did not see a specific impact within the older group. This lack of association in late adulthood may be related to the fact that the older adults had an EAG and $\mathrm{HbA1C}$ in the normal range. The overall negative association is consistent with literature showing that higher glucose ingestion is associated with negative changes in brain activity and connectivity [69], or that glucose fluctuations are linked to disrupted brain functional architecture and cognitive impairment [70]. This may also reflect that the impact of glycemia on brain function and cognition is independent of age.

While this study is among the first to investigate the covariation between different types of brain functional and structural features and a large series of behavioral and health variables across the adult lifespan, we must acknowledge specific limitations. First, our analyses were based on cross-sectional samples and not longitudinal data. As discussed by the STAC-r model, investigating the rate of within-subject cognitive change is essential to understand and identify brain integrity preservation versus compensatory mechanisms which may support preserved cognition in older adults [34]. To our knowledge, there is not yet any large longitudinal cohorts from older - or even youngeradults available to conduct sCCAs in a longitudinal fashion and identify features that predict future brain and cognitive preservation. Future studies should also investigate the impact of structured physical or cognitive interventions on brain-behavior covariations in late adulthood. Second, it will be important to test the reproducibility of our findings in a larger independent sample. It would also be interesting to investigate the impact of pathologic aging on the findings, although this might be challenging as clinical populations may not be able to complete all tasks in one session, as done in the current study. It is also likely that further differences between other subgroups could be revealed, especially in late adulthood (e.g., between participants aged 50-60 years-old and participants above 60, retired versus actively working). Such subgroups could also help identify and improve understanding of the origin of preserved cognition in late adulthood, including the role of neurobiology, compensatory processes, or a combination of both [34]. However, our sample size was too small to statistically test such differences. Lastly, we focused on specific tasks covering major cognitive functions and specific brain measures ( $\mathrm{FC}$ and activation). Future studies should test other fMRI tasks and other neuroimaging measures (e.g., diffusion, graph theory) to determine their unique patterns of covariation with behavior, health and demographic characteristics.

The current study reinforces the importance of accounting for age in multivariate approaches when investigating the link between behavior and brain features, and confirms its non-linear impact across the lifespan. Our findings also highlight the complex interaction of 1) brain structure with age and variables related to stress and general well-being; and 2) brain activation with functional connectivity in their relationship with age, such that increased connectivity in older age may be used to compensate for the loss of brain activation in the networks supporting higher-order cognitive function. In summary, these data further underscore the need to use more multimodal approaches to investigate the impact of healthy aging on behavior, overall health and brain network organization.

\section{MATERIALS AND METHODS}

\section{Participants}

We recruited a total of 59 healthy individuals and divided them into two age groups: A younger adult group of 29 participants (mean age $(\mathrm{SD})=25.6$ (3.4) years, age range: $20.2-32.8$ years; 16 females) and an older adult group of 30 participants (mean age (SD): 62.5 (4.7) years, age range: 55.4-73.5 years; 19 females). The age cut-off for the older group (i.e., 55 years old minimum) was based on the age criterion used for the Alzheimer's Disease Neuroimaging Initiative (ADNI) [71, 72]. Exclusion criteria included any chronic medical illness affecting central nervous system function, any neurological or psychiatric disorder, acute intercurrent illness, pregnancy, history of head trauma, current substance use disorder, and presence of any ferrous metal implant which may interfere with the MRI data acquisition. The study was approved by the Institutional Review Board for Research with Human Subjects at Boys Town National Research Hospital. Each participant provided written informed consent, and all participants completed the same protocol.

\section{Non-imaging data set}

On the day of the scan, participants completed questionnaires and cognitive tests providing demographic information, personal and family medical history, IQ, cognitive scores, and physical activity. They also provided samples of saliva and blood after the MRI scan in order to extract their level of testosterone and estradiol, $\mathrm{HbAlc}$ and estimated average glucose (see detail in Supplementary Material). These variables were selected based on previous studies $[7,8]$. Variables that were highly correlated $(r>0.85)$ 
or had low inter-subject variability (such as smoking habit, or family history of psychiatric disorders) were not included. A total of 59 variables were extracted and are detailed in Supplementary Table 5.

\section{MRI data acquisition}

Participants were scanned on a 3T Siemens Prisma scanner using a 64-channel head coil. Anatomical and functional acquisitions were similar for all participants and adapted from the sequence parameters provided by the Human Connectome Project (HCP) [73]. Structural images were acquired using a T1-weighted, 3D magnetization-prepared rapid gradient-echo (MPRAGE) sequence with the following parameters: Repetition Time $(\mathrm{TR})=2400 \mathrm{~ms}$, Echo Time $(\mathrm{TE})=$ $2.22 \mathrm{~ms}$, Field of View (FOV): $256 \times 256 \mathrm{~mm}$, matrix size: $320 \times 320,0.8 \mathrm{~mm}$ isotropic resolution, Inversion Time $(\mathrm{TI})=1000 \mathrm{~ms}, 8$ degree-flip angle, bandwidth $=$ $220 \mathrm{~Hz} /$ Pixel, echo spacing $=7.5 \mathrm{~ms}$, in-plane acceleration GRAPPA (GeneRalized Autocalibrating Partial Parallel Acquisition) factor 2, total acquisition time $\sim 7$ min. Participants also completed three tasks and one resting-state fMRI scans, using an identical multiband $\mathrm{T}_{2}{ }^{*}$ sequence with the following acquisition parameters: $\mathrm{TR}=800 \mathrm{~ms}, \mathrm{TE}=37 \mathrm{~ms}$, voxel size $=2 \times$ $2 \times 2 \mathrm{~mm}^{3}$, echo spacing $0.58 \mathrm{~ms}$, bandwidth $=2290$ $\mathrm{Hz} /$ Pixel, number of axial slices $=72$, multi-band acceleration factor $=8$. The numbers of volumes collected were: 450 for the n-back task, 375 for the verb generation task, 340 for the scene encoding memory task, and 460 for the resting-state fMRI.

\section{Task fMRI scans: description of the tasks}

Each participant completed a n-back, a SEM and a VG task. These tasks were specifically chosen because: (1) they are associated with cognitive functions among the most commonly investigated in fMRI, and (2) they have been demonstrated as reliable and robust activators of specific brain networks. In short, the n-back fMRI task that we used was developed for the HCP and aimed to activate the network supporting working memory ability [74]. It has a block design incorporating alternating experimental (2-back) and sensorimotor control (0back) conditions. The SEM task was based on the fMRI task developed by Binder et al. known to be a robust activator of bilateral mesial temporal lobe (MTL) structures [75]. This task has a block design incorporating alternating experimental (scene encoding) and control conditions. During the scene encoding condition, the participant was required to identify a given scene as indoor or outdoor from the variety of landscapes or home/office photos presented. This binary judgment ensured attentional engagement and full encoding of the scene. During the control condition, the participant was presented with scrambled pictures divided into two halves and was required to identify if the two halves were identical. Lastly, the VG task aims to activate a network supporting language function. The task has a block design incorporating alternating experimental (verb generation) and control (passive viewing of ' $x x x x$ ') conditions. In the active condition, participants were instructed to covertly generate an action word in response to a viewed object noun presented on a screen. This task has been extensively used in clinical fMRI protocols for epilepsy to localize and lateralize the language network [76, 77]. A detailed description of each task can be further found in Supplementary Material.

In addition, a resting-state fMRI scan was collected during which participants were instructed to remain still and keep their eyes on a fixation cross throughout the scan. For all participants, the resting-state scan was collected before the three tasks in order to avoid cognitive interference from the tasks on the resting-state mental activity.

\section{Structural preprocessing}

In each individual sMRI dataset, we used FreeSurfer image analysis suite (v.6.0) (http://surfer.nmr.mgh.harvard.edu/) to derive 68 cortical thickness measures and 18 subcortical volumetric measures from the Desikan atlas [78]. The outputs were quality controlled using protocols developed by the ENIGMA consortium (http://enigma.ini.usc.edu/). Prior to being entered in further analyses, subcortical volumes were adjusted for variation in intracranial volume.

\section{FMRI preprocessing}

The resting-state and task-based fMRI data were preprocessed using SPM12 and the DPABI Toolbox [79]. For all runs, preprocessing procedures included motion correction to the first volume with rigid-body alignment; co-registration between the functional scans and the anatomical T1-weighted scan; spatial normalization of the functional images into Montreal Neurological Institute (MNI) stereotaxic standard space; and spatial smoothing within the functional mask with a 6-mm at full-width at half-maximum Gaussian kernel. For the functional connectivity analyses only (including the resting-state fMRI data and the 3 task-based fMRI data sets), additional steps were applied for each dataset including linear detrending, regression of motion parameters, their derivatives (24-parameter model, [80]), and the 
scrubbing parameters [81], as well as white matter (WM), and cerebrospinal fluid (CSF) time series (using a component based noise reduction method, 5 principal components [82]).

\section{Subject exclusion and quality control}

A total of six participants were excluded from group analyses because of right hemispheric dominance for language function based on the VG task $(n=2)$, incomplete collection of the fMRI tasks (i.e., one missing task, $n=2$ ), mini-mental state (MMSE) inferior to $25(n=1)$, and excessive head motion during the resting-state fMRI sequence $(n=1)$. The final sample was 27 younger participants (mean age $(\mathrm{std})=25.5$ (3.4) years, 14 females) and 26 older participants (mean age $(\mathrm{std})=62.1(4.5)$ years, 16 females). Demographic and cognitive details on each group are provided in Supplementary Table 4.

\section{FMRI task activation}

For each task, general linear model analyses were implemented using Statistical Parametric Mapping (SPM12). For each task, the preprocessed singleparticipant images were analyzed in a similar fashion, using a linear convolution model, with vectors of onset representing the experimental (n-back: 2-back; VG: verb generation; SEM: indoor/outdoor categorization) and the control condition (n-back: 0-back; VG: passive viewing of "xxxxx"; SEM: scramble pictures). In each participant, images were produced for the active versus control contrast. In each model, the six movement parameters were entered as nuisance covariates. Serial correlations were removed using an AR(1) model. A high-pass filter (128 seconds) was applied to remove low-frequency noise. At the group level, brain regions activated were identified using a random-effects onesample $t$-test of the single-participant contrast images for each task. Age and sex were entered as covariates. The statistical threshold was set up at a height threshold of a minimum of $\mathrm{T}>3.3$ at the whole brain level, which refers to a maximum $p<0.001$ at the voxel level, and cluster size $>50$ voxels. Furthermore, at the cluster level, activation peaks were only selected if they had a $\mathrm{T}$-value $>4.5$. The threshold was adapted for each task as they differ in statistical power (detail in Supplementary Material, Supplementary Tables 6-8). Using the above parameters, for the n-back task, we identified: 12 regions localized in the dorsolateral prefrontal cortex (dlPFC), inferior parietal lobule, supplementary motor area, precuneus, thalamus and cerebellum (Supplementary Table 6 and Supplementary Figure 2). For the VG task, we identified 8 regions localized in the left inferior frontal gyrus, supplementary motor area, inferior parietal lobe, left hippocampus, and cerebellum (Supplementary Table 8, Supplementary Figure 3A). For the SEM task, we identified 9 regions localized in the fusiform and lingual gyri, retrosplenial cortex, orbitofrontal cortex, and lateral occipital cortex (Supplementary Table 7, Supplementary Figure 3B). For each task, the regions identified were consistent with those expected based on previous studies $[7,76,83-85]$.

\section{Extraction of the activation peaks}

Using the Marsbar toolbox, we created 4-mm radius spherical volumes-of-interest (VOIs) centered on each group peak coordinates of each network node and extracted the mean beta values for each respective contrast in each individual. In total, 29 activation variables per individual were entered in the sCCAs. Effect sizes for each peak activation measure between younger and older groups are reported in Supplementary Figure 4.

\section{Independent component analysis}

We conducted a group-based Independent Component Analysis (ICA) to simultaneously identify the major brain networks across the four functional runs because this approach: (a) is fully data-driven (i.e., does not require a priori seeds), (b) has been widely demonstrated as among the most robust approaches to artifacts with minimal assumptions, and (c) allows the identification of cortical, subcortical and cerebellar networks [86]. Group ICA of fMRI Toolbox (GIFT, https://trendscenter.org/software/, version 3.0c) was used to extract spatially independent components (ICs) across all the fMRI datasets and all the participants [87, 88]. The fMRI data from the three tasks and the restingstate from all participants were concatenated into a single dataset and reduced using two stages of principal component analysis (PCA) [87]. We extracted 20 ICs by using Infomax algorithm [89]. The Infomax algorithm generated a spatial map and a time course of the source signal changes for each IC. This analysis was repeated 20 times using ICASSO for assessing the repeatability of ICs [90] and the 20 most reliable components were identified as the final group-level components. IC's time courses and spatial maps were back-reconstructed for each participant using Group information guided (GIG)-ICA [91] implemented in the GIFT Toolbox. GIG-ICA was chosen because it has been shown as more sensitive to group differences [92]. Finally, the 20 ICs were evaluated to identify functionally relevant brain networks. The criteria for identifying the networks were: (1) the peak clusters of a network should be in the grey matter, and (2) there should be minimal overlap with known vascular, susceptibility, ventricular and edge regions. Following 
this selection procedure, 11 networks were selected for functional connectivity analyses (Figure 5).

\section{Functional connectivity analyses}

Upon completing the group ICA and the selection of the 11 networks, we extracted FNC correlations for each pair of networks and within each dataset separately (each of the three tasks and the restingstate), using the GIFT Toolbox. Before FNC computation, subject-specific time courses were detrended and despiked using 3dDespike (AFNI, 1995), then filtered using a fifth-order Butterworth low-pass filter with a high frequency cutoff of 0.15 $\mathrm{Hz}$. This resulted in $55 \mathrm{FNC}$ measures per dataset and per subject. Effect sizes for each FNC measure between younger and older groups are reported in Supplementary Figure 4.

\section{Sparse canonical correlation analyses}

We conducted sCCAs to determine the covariation patterns between behavioral features with sMRI and with fMRI neuroimaging measures.

We used an sCCA approach with an L1-norm penalty [35], using a MATLAB script available online [93]. To do this, the sCCA specifies linear combinations (pairs of canonical variates) of variables in the behavioral- health dataset and variables in the neuroimaging dataset that best express the maximal correlation (i.e., canonical correlation) between the two datasets. The correlations between the canonical variates are the canonical correlations. To achieve this, the algorithm groups variables from either side into component pairs/dimensions, which are referred to as modes in the present paper. Instead of a classic (non-sparse) canonical correlation analysis, we conducted sCCA because this analysis (a) permits the inclusion of more features than observations and (b) allows stronger inferences regarding the contribution of individual variables (for similar approaches, see [7, 9, 55]; also see reviews by Zhuang et al. [36], Wang, Smallwood [94]).

Prior to being entered into the sCCAs, both imaging and behavioral-health variables were z-standardized. The neuroimaging measures were combined into two datasets: the cortical thickness and subcortical volumes on the one hand (total $n=86$, referred to as the "sMRI dataset"), and the activation-related betas from each task and FNC variables during each fMRI run on the other hand (total $n=249$, referred to as the "fMRI dataset"). The non-imaging measures included 59 variables listed in Supplementary Table 5. For each analysis (i.e., non-imaging versus sMRI dataset, and non-imaging versus fMRI dataset), we selected the optimal sparse criteria combination based on the parameters that corresponded to the values of the model

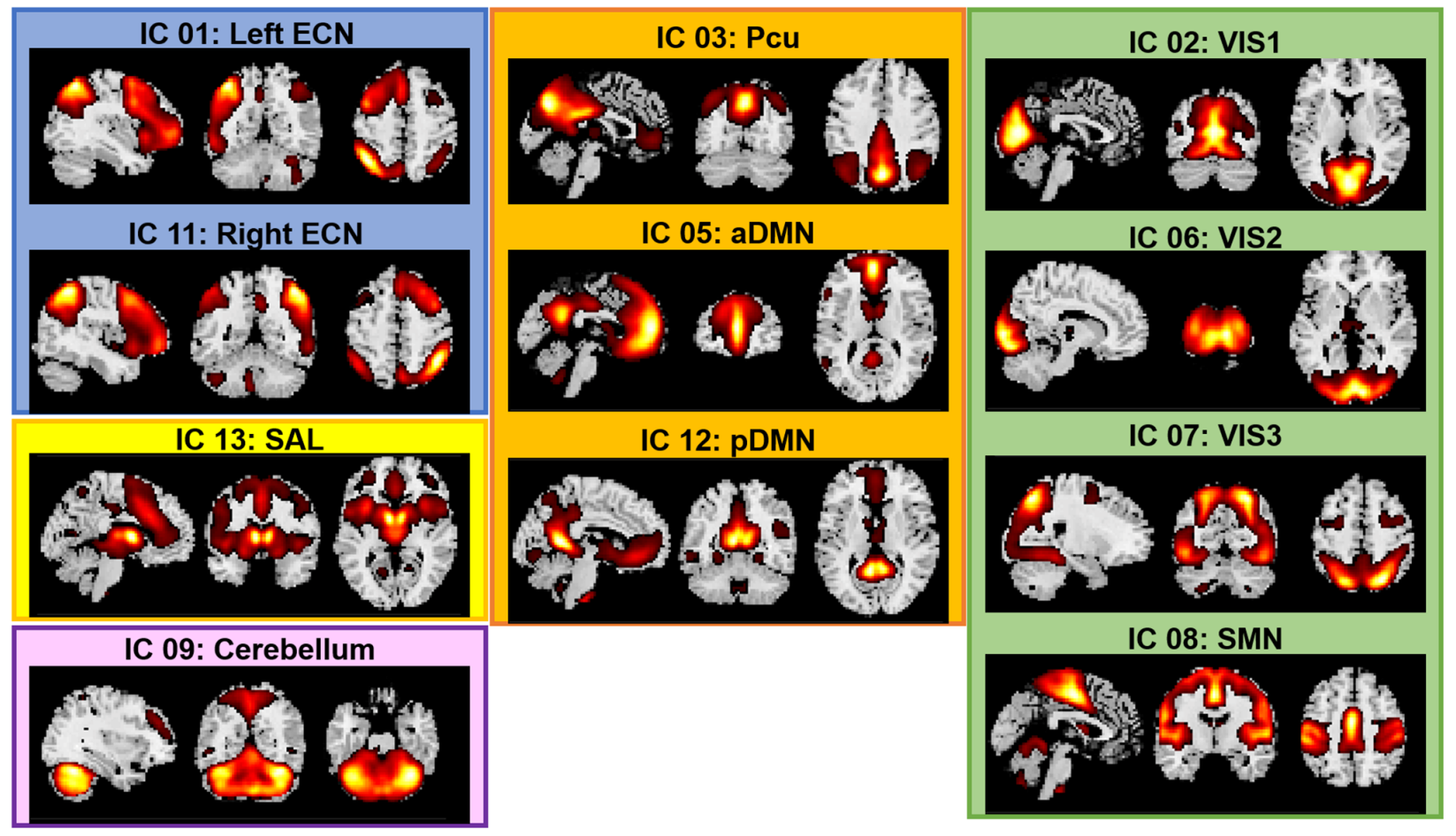

Figure 5. Spatial maps of the 11 networks identified across fMRI sessions and participants. Abbreviations: ECN: Executive Control Network; DMN: Default Mode Network; SAL: Salience Network; SMN: Sensorimotor Network; VIS: Visual Network; a: anterior; p: posterior; Pcu: Precuneus. 
that maximized the sCCA correlation value. We then computed the optimal sCCA model and determined its significance using permutations. Accordingly, the nonimaging dataset was permuted 5,000 times before undergoing the exact same analysis as the original data. The $P$-value was defined as the number of permutations that resulted in a higher correlation than the original data divided by the total number of permutations. Thus, the $P$-value is explicitly corrected for multiple testing as it is compared against the null distribution of maximal correlation values across all estimated sCCAs. The threshold for statistical significance for each analysis was set at $P<0.05$ after 5,000 permutations. When the sCCA was significant, we investigated the contribution weight of each variable in the whole group, and in each subgroup separately (on both the imaging and behavioral data sets). To do so, we computed Pearson's correlations between each variable and the mode of the opposing pattern (that is, each non-imaging variable to mode of the neuroimaging dataset and vice versa).

\section{Subsidiary analyses}

To assess whether our overall results were robust, we 1) performed leave-one out (LOO) analysis for every participant; 2) computed a redundancy-reliability score (Moser's RR-score) for each overall sample sCCA [42]. The RR-score is a measure of the stability of the variable-to-variate correlations and indicates whether results can be expected to be reliable independent of sample composition. The RR-score is based on a training-test set approach and essentially measures whether test sets have similar associations between variables and variates, whereby results with high RRscores can be assumed to be truly carried by the entire sample and not to be dependent on a specific subset of the population that may not be reliably reproduced if one were to replicate the study. It ranges between 0 and 1 ; a value of 0 indicates no correlation between the canonical correlations generated from the randomly resampled 5000 test-sets created during the reliability analyses and a value of 1 indicates complete agreement. In the present study, 5,000 splits of training and test sets were performed in order to calculate the mean RR-score. The RR-score is thus an index of similarity between all testsets. RR-scores that are close to 1 indicate that a test-set would have yielded very similar results as the mean of test-sets. We report the median RR-score and the standard-deviation for each overall sCCA. And 3) We randomly split the sample in half 5,000 times (creating a training and a test sets), performed an sCCA on each of these training sets and then applied the identified weights to the other half of the sample (the test set). This allowed us to gain information on whether sCCA derived correlations in this study were likely to be dependent on this specific sample or not.
The code to compute the Moser's RR score is available at: $\quad$ https://github.com/domamo/Matlab-code-andexample-to-calculate-RR-score-as-related-to-Moser-et$\underline{\text { al-2018. }}$.

\section{AUTHOR CONTRIBUTIONS}

GED and TWW contributed to conception and design of the study. NH and JAK collected the data and organized the dataset. GED, AW and DAM performed the statistical analyses. GED wrote the first draft of the manuscript. GED and DAM wrote sections of the manuscript. All authors contributed to manuscript revision, read, and approved the submitted version.

\section{CONFLICTS OF INTEREST}

The authors declare no conflicts of interest related to this study.

\section{FUNDING}

Funding for this work was provided by the National Institute on Aging (R03AG064001); the National Institute of Mental Health (R01MH116782, RF1MH117032, R01MH118013); the National Institute on Drug Abuse (R01DA047828).

\section{REFERENCES}

1. Alnæs D, Kaufmann $T$, Marquand AF, Smith SM, Westlye LT. Patterns of sociocognitive stratification and perinatal risk in the child brain. Proc Natl Acad Sci U S A. 2020; 117:12419-27.

https://doi.org/10.1073/pnas.2001517117 PMID: $\underline{32409600}$

2. Calderón-Garcidueñas L, Engle R, Mora-Tiscareño A, Styner M, Gómez-Garza G, Zhu H, Jewells V, TorresJardón R, Romero L, Monroy-Acosta ME, Bryant C, González-González LO, Medina-Cortina H, D'Angiulli A. Exposure to severe urban air pollution influences cognitive outcomes, brain volume and systemic inflammation in clinically healthy children. Brain Cogn. 2011; 77:345-55. https://doi.org/10.1016/i.bandc.2011.09.006 PMID:22032805

3. Grasby KL, Jahanshad N, Painter JN, Colodro-Conde L, Bralten J, Hibar DP, Lind PA, Pizzagalli F, Ching CRK, McMahon MAB, Shatokhina N, Zsembik LCP, Thomopoulos SI, et al, and Alzheimer's Disease Neuroimaging Initiative, and CHARGE Consortium, and EPIGEN Consortium, and IMAGEN Consortium, and SYS Consortium, and Parkinson's Progression Markers Initiative, and Enhancing Neurolmaging 
Genetics through Meta-Analysis Consortium (ENIGMA)-Genetics working group. The genetic architecture of the human cerebral cortex. Science. 2020; 367:eaay6690.

https://doi.org/10.1126/science.aay6690 PMID: $\underline{32193296}$

4. Hair NL, Hanson JL, Wolfe BL, Pollak SD. Association of Child Poverty, Brain Development, and Academic Achievement. JAMA Pediatr. 2015; 169:822-9. https://doi.org/10.1001/jamapediatrics.2015.1475 PMID:26192216

5. Lees $B$, Aguinaldo L, Squeglia LM, Infante MA, Wade NE, Hernandez Mejia M, Jacobus J. Parental Family History of Alcohol Use Disorder and Neural Correlates of Response Inhibition in Children From the Adolescent Brain Cognitive Development (ABCD) Study. Alcohol Clin Exp Res. 2020; 44:1234-44. https://doi.org/10.1111/acer.14343 PMID:32333792

6. Noble KG, Houston SM, Brito $\mathrm{NH}$, Bartsch $\mathrm{H}$, Kan E, Kuperman JM, Akshoomoff N, Amaral DG, Bloss CS, Libiger O, Schork NJ, Murray SS, Casey BJ, et al. Family income, parental education and brain structure in children and adolescents. Nat Neurosci. 2015; 18:773-8.

https://doi.org/10.1038/nn.3983

PMID:25821911

7. Moser DA, Doucet GE, Ing A, Dima D, Schumann G, Bilder RM, Frangou S. An integrated brain-behavior model for working memory. Mol Psychiatry. 2018; 23:1974-80.

https://doi.org/10.1038/mp.2017.247 PMID:29203849

8. Smith SM, Nichols TE, Vidaurre D, Winkler AM, Behrens TE, Glasser MF, Ugurbil K, Barch DM, Van Essen DC, Miller KL. A positive-negative mode of population covariation links brain connectivity, demographics and behavior. Nat Neurosci. 2015; 18:1565-7.

https://doi.org/10.1038/nn.4125

PMID:26414616

9. Moser DA, Doucet GE, Lee WH, Rasgon A, Krinsky $H$, Leibu $E$, Ing A, Schumann G, Rasgon N, Frangou S. Multivariate Associations Among Behavioral, Clinical, and Multimodal Imaging Phenotypes in Patients With Psychosis. JAMA Psychiatry. 2018; 75:386-95. https://doi.org/10.1001/jamapsychiatry.2017.4741 PMID:29516092

10. Modabbernia A, Janiri D, Doucet GE, Reichenberg A, Frangou S. Multivariate Patterns of Brain-BehaviorEnvironment Associations in the Adolescent Brain and Cognitive Development Study. Biol Psychiatry. 2021; 89:510-20. https://doi.org/10.1016/j.biopsych.2020.08.014 PMID: $\underline{3109338}$

11. Modabbernia A, Reichenberg A, Ing A, Moser DA, Doucet GE, Artiges E, Banaschewski T, Barker GJ, Becker A, Bokde ALW, Quinlan EB, Desrivières S, Flor $\mathrm{H}$, et al, and IMAGEN Consortium. Linked patterns of biological and environmental covariation with brain structure in adolescence: a population-based longitudinal study. Mol Psychiatry. 2021; 26:4905-18. https://doi.org/10.1038/s41380-020-0757-x PMID: $\underline{32444868}$

12. Chenausky K, Kernbach J, Norton A, Schlaug G. White Matter Integrity and Treatment-Based Change in Speech Performance in Minimally Verbal Children with Autism Spectrum Disorder. Front Hum Neurosci. 2017; 11:175.

https://doi.org/10.3389/fnhum.2017.00175 PMID:28424605

13. Drysdale AT, Grosenick L, Downar J, Dunlop K, Mansouri F, Meng Y, Fetcho RN, Zebley B, Oathes DJ, Etkin A, Schatzberg AF, Sudheimer K, Keller J, et al. Resting-state connectivity biomarkers define neurophysiological subtypes of depression. Nat Med. 2017; 23:28-38.

https://doi.org/10.1038/nm.4246

PMID:27918562

14. Adhikari BM, Hong LE, Sampath H, Chiappelli J, Jahanshad N, Thompson PM, Rowland LM, Calhoun VD, Du X, Chen S, Kochunov P. Functional network connectivity impairments and core cognitive deficits in schizophrenia. Hum Brain Mapp. 2019; 40:4593-605.

https://doi.org/10.1002/hbm.24723

PMID:31313441

15. Doucet GE, Moser DA, Luber MJ, Leibu E, Frangou S. Baseline brain structural and functional predictors of clinical outcome in the early course of schizophrenia. Mol Psychiatry. 2020; 25:863-72.

https://doi.org/10.1038/s41380-018-0269-0 PMID:30283030

16. Lin SJ, Vavasour I, Kosaka B, Li DKB, Traboulsee A, MacKay A, McKeown MJ. Education, and the balance between dynamic and stationary functional connectivity jointly support executive functions in relapsing-remitting multiple sclerosis. Hum Brain Mapp. 2018; 39:5039-49.

https://doi.org/10.1002/hbm.24343

PMID:30240533

17. Miller KL, Alfaro-Almagro F, Bangerter NK, Thomas DL, Yacoub E, Xu J, Bartsch AJ, Jbabdi S, Sotiropoulos SN, Andersson JL, Griffanti L, Douaud G, Okell TW, et al. Multimodal population brain imaging in the UK Biobank prospective epidemiological study. Nat Neurosci. 2016; 19:1523-36. 
https://doi.org/10.1038/nn.4393

PMID:27643430

18. Hedman AM, van Haren NE, Schnack HG, Kahn RS, Hulshoff Pol HE. Human brain changes across the life span: a review of 56 longitudinal magnetic resonance imaging studies. Hum Brain Mapp. 2012; 33:19872002.

https://doi.org/10.1002/hbm.21334

PMID:21915942

19. Meunier D, Achard S, Morcom A, Bullmore E. Agerelated changes in modular organization of human brain functional networks. Neuroimage. 2009; 44:715-23.

https://doi.org/10.1016/j.neuroimage.2008.09.062 PMID:19027073

20. Damoiseaux JS. Effects of aging on functional and structural brain connectivity. Neuroimage. 2017; 160:32-40.

https://doi.org/10.1016/i.neuroimage.2017.01.077 PMID:28159687

21. Dima D, Modabbernia A, Papachristou E, Doucet GE, Agartz I, Aghajani M, Akudjedu TN, Albajes-Eizagirre A, Alnaes D, Alpert KI, Andersson M, Andreasen NC, Andreassen OA, et al, and Karolinska Schizophrenia Project (KaSP). Subcortical volumes across the lifespan: Data from 18,605 healthy individuals aged 390 years. Hum Brain Mapp. 2022; 43:452-69.

https://doi.org/10.1002/hbm.25320

PMID:33570244

22. Frangou $S$, Modabbernia A, Williams SCR, Papachristou E, Doucet GE, Agartz I, Aghajani M, Akudjedu TN, Albajes-Eizagirre A, Alnaes D, Alpert KI, Andersson M, Andreasen NC, et al, and Karolinska Schizophrenia Project (KaSP). Cortical thickness across the lifespan: Data from 17,075 healthy individuals aged 3-90 years. Hum Brain Mapp. 2022; 43:431-51. https://doi.org/10.1002/hbm.25364 PMID:33595143

23. Doucet GE, Labache L, Thompson PM, Joliot $M$, Frangou S, and Alzheimer's Disease Neuroimaging Initiative. Atlas55+: Brain Functional Atlas of RestingState Networks for Late Adulthood. Cereb Cortex. 2021; 31:1719-31.

https://doi.org/10.1093/cercor/bhaa321

PMID:33188411

24. Betzel RF, Byrge L, He Y, Goñi J, Zuo XN, Sporns O. Changes in structural and functional connectivity among resting-state networks across the human lifespan. Neuroimage. 2014; 102:345-57. https://doi.org/10.1016/i.neuroimage.2014.07.067 PMID:25109530

25. Varangis E, Habeck CG, Razlighi QR, Stern Y. The Effect of Aging on Resting State Connectivity of
Predefined Networks in the Brain. Front Aging Neurosci. 2019; 11:234.

https://doi.org/10.3389/fnagi.2019.00234

PMID:31555124

26. Ferreira LK, Regina AC, Kovacevic N, Martin Mda G, Santos PP, Carneiro Cde G, Kerr DS, Amaro E Jr, Mclntosh AR, Busatto GF. Aging Effects on Whole-Brain Functional Connectivity in Adults Free of Cognitive and Psychiatric Disorders. Cereb Cortex. 2016; 26:3851-65. https://doi.org/10.1093/cercor/bhv190 PMID:26315689

27. Kwak S, Park S, Kim J, Park S, Lee JY. Multivariate neuroanatomical correlates of behavioral and psychological symptoms in dementia and the moderating role of education. Neuroimage Clin. 2020; 28:102452.

https://doi.org/10.1016/j.nicl.2020.102452

PMID:33070098

28. Liao J, Zhu Y, Zhang M, Yuan H, Su MY, Yu X, Wang H. Microstructural white matter abnormalities independent of white matter lesion burden in amnestic mild cognitive impairment and early Alzheimer disease among Han Chinese elderly. Alzheimer Dis Assoc Disord. 2010; 24:317-24. https://doi.org/10.1097/WAD.0b013e3181df1c7b PMID:20592582

29. Tomasi D, Wang R, Wang GJ, Volkow ND. Functional connectivity and brain activation: a synergistic approach. Cereb Cortex. 2014; 24:2619-29.

https://doi.org/10.1093/cercor/bht119 PMID:23645721

30. Tomasi D, Volkow ND. Association Between Brain Activation and Functional Connectivity. Cereb Cortex. 2019; 29:1984-96.

https://doi.org/10.1093/cercor/bhy077 PMID:29668865

31. La C, Mossahebi P, Nair VA, Bendlin BB, Birn R, Meyerand ME, Prabhakaran V. Age-Related Changes in Inter-Network Connectivity by Component Analysis. Front Aging Neurosci. 2015; 7:237. https://doi.org/10.3389/fnagi.2015.00237 PMID:26733864

32. Meunier D, Stamatakis EA, Tyler LK. Age-related functional reorganization, structural changes, and preserved cognition. Neurobiol Aging. 2014; 35:42-54. https://doi.org/10.1016/i.neurobiolaging.2013.07.003 PMID:23942392

33. Kennedy KM, Rodrigue KM, Bischof GN, Hebrank AC, Reuter-Lorenz PA, Park DC. Age trajectories of functional activation under conditions of low and high processing demands: an adult lifespan fMRI study of the aging brain. Neuroimage. 2015; 104:21-34. 
https://doi.org/10.1016/j.neuroimage.2014.09.056 PMID:25284304

34. Reuter-Lorenz PA, Park DC. How does it STAC up? Revisiting the scaffolding theory of aging and cognition. Neuropsychol Rev. 2014; 24:355-70. https://doi.org/10.1007/s11065-014-9270-9 PMID:25143069

35. Witten DM, Tibshirani R, Hastie T. A penalized matrix decomposition, with applications to sparse principal components and canonical correlation analysis. Biostatistics. 2009; 10:515-34.

https://doi.org/10.1093/biostatistics/kxp008 PMID:19377034

36. Zhuang X, Yang Z, Cordes D. A technical review of canonical correlation analysis for neuroscience applications. Hum Brain Mapp. 2020; 41:3807-33.

https://doi.org/10.1002/hbm.25090

PMID: 32592530

37. Klami A, Virtanen S, Kaski S. Bayesian Canonical correlation analysis. J Mach Learn Res. 2013; 14:9651003.

38. Moser DA, Dricu M, Wiest R, Schüpbach L, Aue T. Social optimism biases are associated with cortical thickness. Soc Cogn Affect Neurosci. 2020; 15:745-54. https://doi.org/10.1093/scan/nsaa095 PMID:32685967

39. Daffner KR. Promoting successful cognitive aging: a comprehensive review. J Alzheimers Dis. 2010; 19:1101-22.

https://doi.org/10.3233/JAD-2010-1306 PMID:20308777

40. Reuter-Lorenz PA, Cappell KA. Neurocognitive Aging and the Compensation Hypothesis. Curr Dir Psychol Sci. 2008; 17:177-82.

https://doi.org/10.1111/j.1467-8721.2008.00570.x

41. Park DC, Reuter-Lorenz P. The adaptive brain: aging and neurocognitive scaffolding. Annu Rev Psychol. 2009; 60:173-96.

https://doi.org/10.1146/annurev.psych.59.103006.09 $\underline{3656}$

PMID:19035823

42. Moser DA. Matlab code and example to calculate RRscore as related to Moser et al 2018. 2018. https://github.com/domamo/Matlab-code-andexample-to-calculate-RR-score-as-related-to-Moseret-al-2018.

43. Baddeley A. Working memory: theories, models, and controversies. Annu Rev Psychol. 2012; 63:1-29. https://doi.org/10.1146/annurev-psych-120710100422

PMID:21961947
44. Yaple ZA, Stevens WD, Arsalidou M. Meta-analyses of the n-back working memory task: fMRI evidence of agerelated changes in prefrontal cortex involvement across the adult lifespan. Neuroimage. 2019; 196:16-31.

https://doi.org/10.1016/i.neuroimage.2019.03.074 PMID:30954708

45. Salehi M, Greene AS, Karbasi A, Shen X, Scheinost D, Constable RT. There is no single functional atlas even for a single individual: Functional parcel definitions change with task. Neuroimage. 2020; 208:116366. https://doi.org/10.1016/j.neuroimage.2019.116366 PMID:31740342

46. Salehi M, Karbasi A, Barron DS, Scheinost D, Constable RT. Individualized functional networks reconfigure with cognitive state. Neuroimage. 2020; 206:116233.

https://doi.org/10.1016/j.neuroimage.2019.116233 PMID:31574322

47. Finn ES, Shen X, Scheinost D, Rosenberg MD, Huang J, Chun MM, Papademetris X, Constable RT. Functional connectome fingerprinting: identifying individuals using patterns of brain connectivity. Nat Neurosci. 2015; 18:1664-71. https://doi.org/10.1038/nn.4135 PMID:26457551

48. Varangis E, Razlighi Q, Habeck CG, Fisher Z, Stern Y. Between-network Functional Connectivity Is Modified by Age and Cognitive Task Domain. J Cogn Neurosci. 2019; 31:607-22.

https://doi.org/10.1162/jocn a 01368

PMID:30605005

49. Doucet G, Naveau M, Petit L, Delcroix N, Zago L, Crivello F, Jobard G, Tzourio-Mazoyer N, Mazoyer B, Mellet $\mathrm{E}$, Joliot $\mathrm{M}$. Brain activity at rest: a multiscale hierarchical functional organization. J Neurophysiol. 2011; 105:2753-63.

https://doi.org/10.1152/jn.00895.2010

PMID:21430278

50. Doucet G, Naveau M, Petit L, Zago L, Crivello F, Jobard G, Delcroix N, Mellet E, Tzourio-Mazoyer N, Mazoyer $B$, Joliot M. Patterns of hemodynamic low-frequency oscillations in the brain are modulated by the nature of free thought during rest. Neuroimage. 2012; 59:3194-200.

https://doi.org/10.1016/i.neuroimage.2011.11.059 PMID:22155378

51. Fox MD, Snyder AZ, Vincent JL, Corbetta M, Van Essen DC, Raichle ME. The human brain is intrinsically organized into dynamic, anticorrelated functional networks. Proc Natl Acad Sci U S A. 2005; 102:9673-8. https://doi.org/10.1073/pnas.0504136102 PMID:15976020 
52. Whitfield-Gabrieli S, Thermenos HW, Milanovic S, Tsuang MT, Faraone SV, McCarley RW, Shenton ME, Green Al, Nieto-Castanon A, LaViolette P, Wojcik J, Gabrieli JD, Seidman $\sqcup$. Hyperactivity and hyperconnectivity of the default network in schizophrenia and in first-degree relatives of persons with schizophrenia. Proc Natl Acad Sci U S A. 2009; 106:1279-84.

https://doi.org/10.1073/pnas.0809141106

PMID:19164577

53. Carstensen LL, Turan B, Scheibe S, Ram N, ErsnerHershfield H, Samanez-Larkin GR, Brooks KP, Nesselroade JR. Emotional experience improves with age: evidence based on over 10 years of experience sampling. Psychol Aging. 2011; 26:21-33.

https://doi.org/10.1037/a0021285

PMID:20973600

54. Lydon-Staley DM, Kuehner C, Zamoscik V, Huffziger S, Kirsch P, Bassett DS. Repetitive negative thinking in daily life and functional connectivity among default mode, fronto-parietal, and salience networks. Transl Psychiatry. 2019; 9:234.

https://doi.org/10.1038/s41398-019-0560-0

PMID:31534117

55. Moser DA, Dricu M, Kotikalapudi R, Doucet GE, Aue T. Reduced network integration in default mode and executive networks is associated with social and personal optimism biases. Hum Brain Mapp. 2021; 42:2893-906.

https://doi.org/10.1002/hbm.25411

PMID:33755272

56. Janiri D, Moser DA, Doucet GE, Luber MJ, Rasgon A, Lee WH, Murrough JW, Sani G, Eickhoff SB, Frangou S. Shared Neural Phenotypes for Mood and Anxiety Disorders: A Meta-analysis of 226 Task-Related Functional Imaging Studies. JAMA Psychiatry. 2020; 77:172-9.

https://doi.org/10.1001/jamapsychiatry.2019.3351 PMID:31664439

57. Mather M. The Affective Neuroscience of Aging. Annu Rev Psychol. 2016; 67:213-38.

https://doi.org/10.1146/annurev-psych-122414033540

PMID:26436717

58. Schmaal L, Veltman DJ, van Erp TG, Sämann PG, Frodl T, Jahanshad N, Loehrer E, Tiemeier H, Hofman A, Niessen WJ, Vernooij MW, Ikram MA, Wittfeld K, et al. Subcortical brain alterations in major depressive disorder: findings from the ENIGMA Major Depressive Disorder working group. Mol Psychiatry. 2016; 21:806-12.

https://doi.org/10.1038/mp.2015.69

PMID:26122586

59. Doucet GE, Rasgon N, McEwen BS, Micali N, Frangou S. Elevated Body Mass Index is Associated with
Increased Integration and Reduced Cohesion of Sensory-Driven and Internally Guided Resting-State Functional Brain Networks. Cereb Cortex. 2018; 28:988-97.

https://doi.org/10.1093/cercor/bhx008

PMID:28119342

60. Huerta Cl, Sarkar PR, Duong TQ, Laird AR, Fox PT. Neural bases of food perception: coordinate-based meta-analyses of neuroimaging studies in multiple modalities. Obesity (Silver Spring). 2014; 22:1439-46. https://doi.org/10.1002/oby.20659 PMID:24174404

61. He X, Qin W, Liu Y, Zhang X, Duan Y, Song J, Li K, Jiang $T$, Yu C. Age-related decrease in functional connectivity of the right fronto-insular cortex with the central executive and default-mode networks in adults from young to middle age. Neurosci Lett. 2013; 544:74-9.

https://doi.org/10.1016/j.neulet.2013.03.044 PMID:23583587

62. Luo N, Sui J, Abrol A, Chen J, Turner JA, Damaraju E, Fu Z, Fan L, Lin D, Zhuo C, Xu Y, Glahn DC, Rodrigue $A L$, et al. Structural Brain Architectures Match Intrinsic Functional Networks and Vary across Domains: A Study from $15000+$ Individuals. Cereb Cortex. 2020; 30:5460-70.

https://doi.org/10.1093/cercor/bhaa127 PMID: $\underline{32488253}$

63. Abd Hamid Al, Speck O, Hoffmann MB. Quantitative assessment of visual cortex function with $\mathrm{fMRI}$ at 7 Tesla-test-retest variability. Front Hum Neurosci. 2015; 9:477. https://doi.org/10.3389/fnhum.2015.00477 PMID:26388756

64. Benson NC, Butt OH, Datta R, Radoeva PD, Brainard $\mathrm{DH}$, Aguirre GK. The retinotopic organization of striate cortex is well predicted by surface topology. Curr Biol. 2012; 22:2081-5. https://doi.org/10.1016/i.cub.2012.09.014 PMID:23041195

65. Casanova MF, Trippe J 2nd, Tillquist C, Switala AE. Morphometric variability of minicolumns in the striate cortex of Homo sapiens, Macaca mulatta, and Pan troglodytes. J Anat. 2009; 214:226-34. https://doi.org/10.1111/i.1469-7580.2008.01027.x PMID: 19207984

66. Franco AR, Mannell MV, Calhoun VD, Mayer AR. Impact of analysis methods on the reproducibility and reliability of resting-state networks. Brain Connect. 2013; 3:363-74.

https://doi.org/10.1089/brain.2012.0134 PMID:23705789

67. Li R, Yin S, Zhu X, Ren W, Yu J, Wang P, Zheng Z, Niu YN, Huang $X$, Li J. Linking Inter-Individual Variability in 
Functional Brain Connectivity to Cognitive Ability in Elderly Individuals. Front Aging Neurosci. 2017; 9:385. https://doi.org/10.3389/fnagi.2017.00385 PMID:29209203

68. Mueller S, Wang D, Fox MD, Yeo BT, Sepulcre J, Sabuncu MR, Shafee R, Lu J, Liu H. Individual variability in functional connectivity architecture of the human brain. Neuron. 2013; 77:586-95.

https://doi.org/10.1016/j.neuron.2012.12.028 PMID:23395382

69. van Opstal AM, Hafkemeijer A, van den BergHuysmans AA, Hoeksma M, Blonk C, Pijl H, Rombouts SAR, van der Grond J. Brain activity and connectivity changes in response to glucose ingestion. Nutr Neurosci. 2020; 23:110-7. https://doi.org/10.1080/1028415X.2018.1477538 PMID:29806553

70. Xia W, Luo Y, Chen YC, Chen H, Ma J, Yin X. Glucose Fluctuations Are Linked to Disrupted Brain Functional Architecture and Cognitive Impairment. J Alzheimers Dis. 2020; 74:603-13. https://doi.org/10.3233/JAD-191217 PMID: $\underline{32065795}$

71. Jack CR Jr, Bernstein MA, Fox NC, Thompson $P$, Alexander G, Harvey D, Borowski B, Britson PJ, Whitwell JL, Ward C, Dale AM, Felmlee JP, Gunter JL, et al. The Alzheimer's Disease Neuroimaging Initiative (ADNI): MRI methods. J Magn Reson Imaging. 2008; 27:685-91.

https://doi.org/10.1002/jmri.21049

PMID:18302232

72. Petersen RC, Aisen PS, Beckett LA, Donohue MC, Gamst AC, Harvey DJ, Jack CR Jr, Jagust WJ, Shaw LM, Toga AW, Trojanowski JQ, Weiner MW. Alzheimer's Disease Neuroimaging Initiative (ADNI): clinical characterization. Neurology. 2010; 74:201-9. https://doi.org/10.1212/WNL.0b013e3181cb3e25 PMID:20042704

73. Glasser MF, Smith SM, Marcus DS, Andersson JL, Auerbach EJ, Behrens TE, Coalson TS, Harms MP, Jenkinson M, Moeller S, Robinson EC, Sotiropoulos SN, Xu $\mathrm{J}$, et al. The Human Connectome Project's neuroimaging approach. Nat Neurosci. 2016; 19:1175-87.

https://doi.org/10.1038/nn.4361

PMID:27571196

74. Barch DM, Burgess GC, Harms MP, Petersen SE, Schlaggar BL, Corbetta M, Glasser MF, Curtiss S, Dixit S, Feldt C, Nolan D, Bryant E, Hartley T, et al, and WUMinn HCP Consortium. Function in the human connectome: task-fMRI and individual differences in behavior. Neuroimage. 2013; 80:169-89.

https://doi.org/10.1016/i.neuroimage.2013.05.033 PMID:23684877
75. Binder JR, Bellgowan PS, Hammeke TA, Possing ET, Frost JA. A comparison of two FMRI protocols for eliciting hippocampal activation. Epilepsia. 2005; 46:1061-70.

https://doi.org/10.1111/i.1528-1167.2005.62004.x PMID:16026558

76. Doucet GE, He X, Sperling MR, Sharan A, Tracy JI. From "rest" to language task: Task activation selects and prunes from broader resting-state network. Hum Brain Mapp. 2017; 38:2540-52.

https://doi.org/10.1002/hbm.23539 PMID:28195438

77. Doucet GE, Pustina D, Skidmore C, Sharan A, Sperling $M R$, Tracy JI. Resting-state functional connectivity predicts the strength of hemispheric lateralization for language processing in temporal lobe epilepsy and normals. Hum Brain Mapp. 2015; 36:288-303.

https://doi.org/10.1002/hbm.22628

PMID:25187327

78. Desikan RS, Ségonne F, Fischl B, Quinn BT, Dickerson BC, Blacker D, Buckner RL, Dale AM, Maguire RP, Hyman BT, Albert MS, Killiany RJ. An automated labeling system for subdividing the human cerebral cortex on MRI scans into gyral based regions of interest. Neuroimage. 2006; 31:968-80.

https://doi.org/10.1016/j.neuroimage.2006.01.021 PMID:16530430

79. Yan CG, Wang XD, Zuo XN, Zang YF. DPABI: Data Processing \& Analysis for (Resting-State) Brain Imaging. Neuroinformatics. 2016; 14:339-51. https://doi.org/10.1007/s12021-016-9299-4 PMID:27075850

80. Friston KJ, Williams S, Howard R, Frackowiak RS, Turner R. Movement-related effects in fMRI timeseries. Magn Reson Med. 1996; 35:346-55. https://doi.org/10.1002/mrm.1910350312 PMID:8699946

81. Power JD, Barnes KA, Snyder AZ, Schlaggar BL, Petersen SE. Spurious but systematic correlations in functional connectivity MRI networks arise from subject motion. Neuroimage. 2012; 59:2142-54. https://doi.org/10.1016/j.neuroimage.2011.10.018 PMID:22019881

82. Behzadi Y, Restom K, Liau J, Liu TT. A component based noise correction method (CompCor) for BOLD and perfusion based fMRI. Neuroimage. 2007; 37:90-101. https://doi.org/10.1016/i.neuroimage.2007.04.042 PMID:17560126

83. Owen AM, Herrod NJ, Menon DK, Clark JC, Downey SP, Carpenter TA, Minhas PS, Turkheimer FE, Williams EJ, Robbins TW, Sahakian BJ, Petrides M, Pickard JD. Redefining the functional organization of working 
memory processes within human lateral prefrontal cortex. Eur J Neurosci. 1999; 11:567-74. https://doi.org/10.1046/j.1460-9568.1999.00449.x PMID:10051756

84. Rottschy C, Langner R, Dogan I, Reetz K, Laird AR, Schulz JB, Fox PT, Eickhoff SB. Modelling neural correlates of working memory: a coordinate-based meta-analysis. Neuroimage. 2012; 60:830-46. https://doi.org/10.1016/i.neuroimage.2011.11.050 PMID:22178808

85. Sideman N, Chaitanya G, He X, Doucet G, Kim NY, Sperling MR, Sharan AD, Tracy JI. Task activation and functional connectivity show concordant memory laterality in temporal lobe epilepsy. Epilepsy Behav. 2018; 81:70-8.

https://doi.org/10.1016/i.yebeh.2018.01.027 PMID:29499551

86. Calhoun VD, de Lacy N. Ten Key Observations on the Analysis of Resting-state Functional MR Imaging Data Using Independent Component Analysis. Neuroimaging Clin N Am. 2017; 27:561-79. https://doi.org/10.1016/j.nic.2017.06.012 PMID:28985929

87. Calhoun VD, Adali T, Pearlson GD, Pekar JJ. A method for making group inferences from functional MRI data using independent component analysis. Hum Brain Mapp. 2001; 14:140-51. https://doi.org/10.1002/hbm.1048 PMID:11559959

88. Calhoun VD, Liu J, Adali T. A review of group ICA for fMRI data and ICA for joint inference of imaging, genetic, and ERP data. Neuroimage. 2009; 45:S163-72. https://doi.org/10.1016/i.neuroimage.2008.10.057 PMID:19059344

89. Bell AJ, Sejnowski TJ. An information-maximization approach to blind separation and blind deconvolution. Neural Comput. 1995; 7:1129-59. https://doi.org/10.1162/neco.1995.7.6.1129 PMID:
90. Himberg J, Hyvärinen A, Esposito F. Validating the independent components of neuroimaging time series via clustering and visualization. Neuroimage. 2004; 22:1214-22.

https://doi.org/10.1016/i.neuroimage.2004.03.027 PMID:15219593

91. Du Y, Fan Y. Group information guided ICA for $\mathrm{fMRI}$ data analysis. Neuroimage. 2013; 69:157-97. https://doi.org/10.1016/i.neuroimage.2012.11.008 PMID:23194820

92. Salman MS, Du Y, Lin D, Fu Z, Fedorov A, Damaraju E, Sui J, Chen J, Mayer AR, Posse S, Mathalon DH, Ford JM, Van Erp T, Calhoun VD. Group ICA for identifying biomarkers in schizophrenia: 'Adaptive' networks via spatially constrained ICA show more sensitivity to group differences than spatio-temporal regression. Neuroimage Clin. 2019; 22:101747.

https://doi.org/10.1016/i.nicl.2019.101747 PMID:30921608

93. Ing A, Sämann PG, Chu C, Tay N, Biondo F, Robert G, Jia $T$, Wolfers $T$, Desrivières $S$, Banaschewski $T$, Bokde ALW, Bromberg $U$, Büchel $C$, et al, and IMAGEN Consortium. Identification of neurobehavioural symptom groups based on shared brain mechanisms. Nat Hum Behav. 2019; 3:1306-18. https://doi.org/10.1038/s41562-019-0738-8 PMID:31591521

94. Wang HT, Smallwood J, Mourao-Miranda J, Xia CH, Satterthwaite TD, Bassett DS, Bzdok D. Finding the needle in a high-dimensional haystack: Canonical correlation analysis for neuroscientists. Neuroimage. 2020; 216:116745.

https://doi.org/10.1016/j.neuroimage.2020.116745 PMID: $\underline{32278095}$ 


\section{SUPPLEMENTARY MATERIALS}

\section{Supplementary Materials and Methods}

\section{Non-imaging dataset collection}

Participants completed a series of questionnaires regarding demographic information, medical history for both self and biological family members, and lifetime of experiences. In order to measure their cognitive capacity, they completed the Mini-Mental Status Examination (MMSE), WASI-II [1], and the NIH Toolbox for Adult, including cognition, emotion and motor batteries (https://www.healthmeasures.net/ explore-measurement-systems/nih-toolbox/intro-to-nihtoolbox). Lastly, immediately after the fMRI scan, samples of saliva and blood were collected. From the blood sample, HbAlc and estimated average glucose (EAG) levels were extracted. The saliva sample was analyzed by the University of Nebraska-Lincoln Salivary Bioscience Laboratory to measure the level of estradiol and testosterone. A total of 59 non-imaging variables were extracted and described in Supplementary Table 5.

\section{Task description}

\section{N-back task}

The task was the one developed by the Human Connectome Project (HCP), and has a block design incorporating alternating experimental (2-back) and sensorimotor control (0-back) conditions [2]. At the start of a block, a written cue (lasting 2.5 seconds) informed participants about the type of condition to follow (2-back or 0-back) and the designated target stimulus for the sensorimotor control condition. Four different stimulus types (faces, places, tools and body parts) were presented in separate blocks. Each stimulus was presented for 3 seconds, followed by a $500 \mathrm{~ms}$ inter-stimulus interval. In the 2-back trials participants were asked to respond by pressing a button whenever the current stimulus was the same as the one presented 2 trials back. In the 0-back condition, participants were asked to respond by pressing a button each time they saw the designated stimulus. The run contained 8 blocks of 10 trials, each lasting 35 seconds, and 4 fixation blocks each lasting 15 seconds. Performance features (accuracy and reaction time) were monitored and collected during fMRI scanning.

\section{Verb Generation (VG) task}

This task is known to be a robust activator of the language network [3, 4]. Participants were instructed to covertly generate an action word in response to a viewed concrete noun presented on a screen. Each word was presented for $3 \mathrm{~s}$, within a 30-second block. These blocks were alternated with passive viewing of a central stimulus (\#\#\#\#) in epochs of $30 \mathrm{~s}$ for a total of 5 min.

\section{Scene Encoding Memory (SEM) task}

This task was adapted from the task created by Binder et al. [5] known to be a robust activator of bilateral mesial temporal lobe (MTL) structures. A block-design format was utilized. During the scene encoding condition, the participant was required to identify a given scene as indoor or outdoor from the variety of landscapes or home/office photos presented. This binary judgment ensured attentional engagement and full encoding of the scene. During the control condition, the participant was presented with scrambled pictures divided into two halves, and required to identify if the two halves were identical. This control condition allowed us to subtract the visuo-perceptual and decision-making aspects of task performance. The session contained four blocks of scene encoding and four blocks of the control condition, each block for $28 \mathrm{~s}$ in duration. Each session started and ended with a 28 -second period of blank screen. For each of the scene encoding and control blocks, 8 stimuli (e.g., scene/scrambled picture) were presented, each for $3 \mathrm{~s}$, interleaved with a $0.5 \mathrm{~s}$ of blank screen. The participant discriminated indoor scenes from outdoor scenes (indoor session), and vice versa in the other session (outdoor session), with the order of sessions randomly counterbalanced across participants. $60 \%$ of the stimuli presented during the scene encoding blocks within that session were the targets, and the rest $(40 \%)$ were nontargets; while, $60 \%$ of the stimuli presented during the control blocks contained scrambled pictures (halves) that were identically matched (to be referred to as targets as well), and the other $40 \%$ were not (i.e., nontargets). All visual stimuli were presented only once during the entire study. The participants were instructed to press a button with their index finger for target pictures and another button with their middle finger for non-target pictures. Performance features (accuracy and reaction time) were monitored and collected during fMRI scanning.

\section{Task activation}

N-back task

See Supplementary Table 6 and Supplementary Figure 2.

Verb Generation

See Supplementary Table 8 and Supplementary Figure 3. 
Scene Encoding Memory

See Supplementary Table 7 and Supplementary Figure 3.

\section{Difference in brain activity between younger and older participants}

We computed the effect size for each functional measure (Cohen's D) between younger and older participants (Supplementary Figure 4). For both VG and N-back tasks, older participants showed lower activation, while for the SEM task, older showed higher activation, than the younger participants. For the FNC measures, older participants showed a large majority of lower FC, compared to the younger participants.

\section{Sparse canonical correlation analyses (sCCAs)}

Non-imaging dataset vs. sMRI dataset

See Supplementary Tables 1 and 2.

Non-imaging dataset vs. fMRI dataset

See Supplementary Table 3.

\section{Reliability analyses}

See Supplementary Figure 1.

\section{REFERENCES}

1. Wechsler D. Wechsler Abbreviated Scale of Intelligence | Second Edition. San Antonio, TX: NCS Pearson. 2011.

2. Barch DM, Burgess GC, Harms MP, Petersen SE, Schlaggar BL, Corbetta M, Glasser MF, Curtiss S, Dixit S, Feldt C, Nolan D, Bryant E, Hartley T, et al, and WUMinn HCP Consortium. Function in the human connectome: task-fMRI and individual differences in behavior. Neuroimage. 2013; 80:169-89.

https://doi.org/10.1016/i.neuroimage.2013.05.033 PMID:23684877

3. Doucet GE, He X, Sperling MR, Sharan A, Tracy JI. From "rest" to language task: Task activation selects and prunes from broader resting-state network. Hum Brain Mapp. 2017; 38:2540-52. https://doi.org/10.1002/hbm.23539 PMID:28195438

4. Binder JR, Frost JA, Hammeke TA, Bellgowan PS, Springer JA, Kaufman JN, Possing ET. Human temporal lobe activation by speech and nonspeech sounds. Cereb Cortex. 2000; 10:512-28. https://doi.org/10.1093/cercor/10.5.512 PMID:10847601

5. Binder JR, Bellgowan PS, Hammeke TA, Possing ET, Frost JA. A comparison of two FMRI protocols for eliciting hippocampal activation. Epilepsia. 2005; 46:1061-70.

https://doi.org/10.1111/j.1528-1167.2005.62004.x PMID:16026558 
Supplementary Figures

\section{Distribution of Mosers RR-Scores}

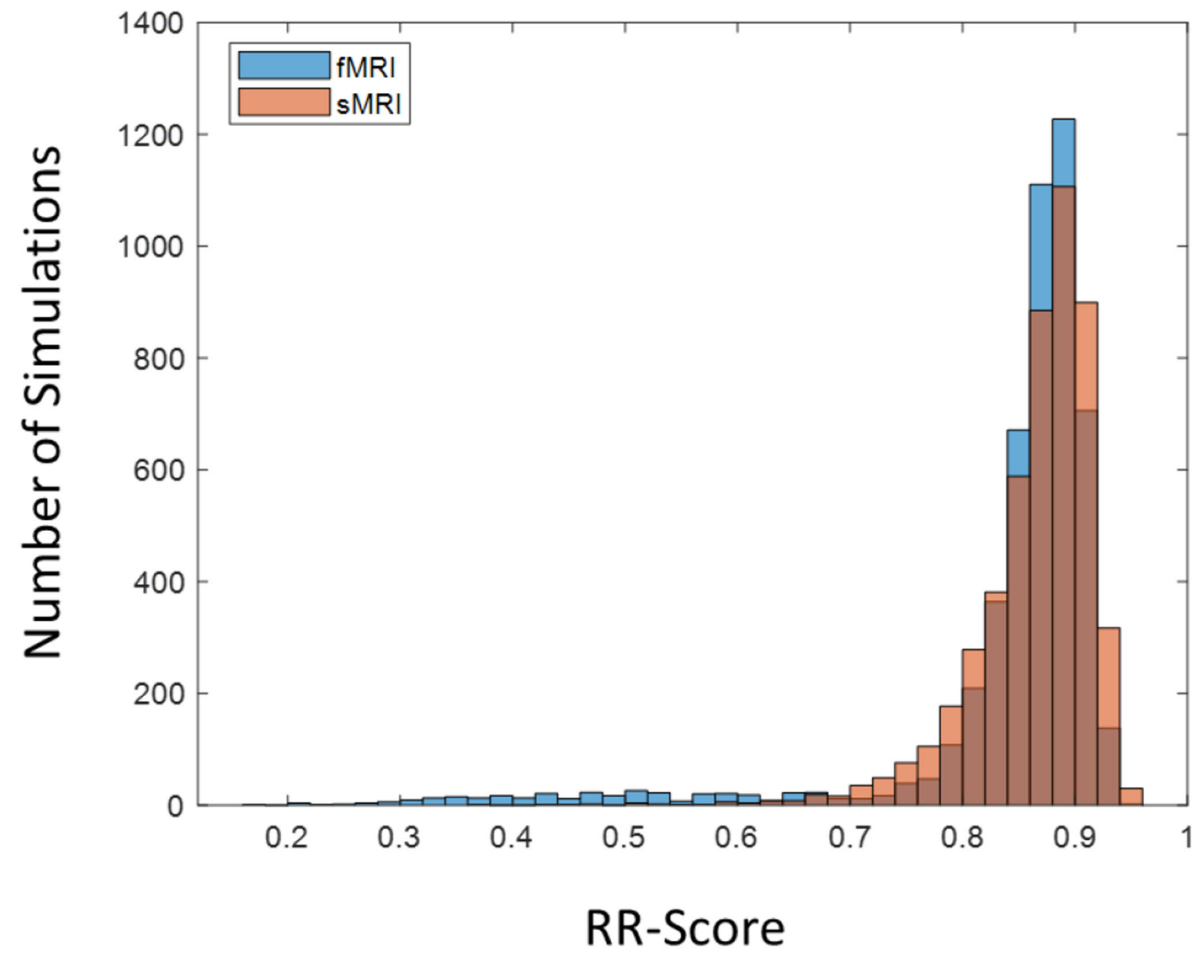

Supplementary Figure 1. Distribution of the redundancy-reliability (RR) score for each sCCA, over the 5,000 simulations. 

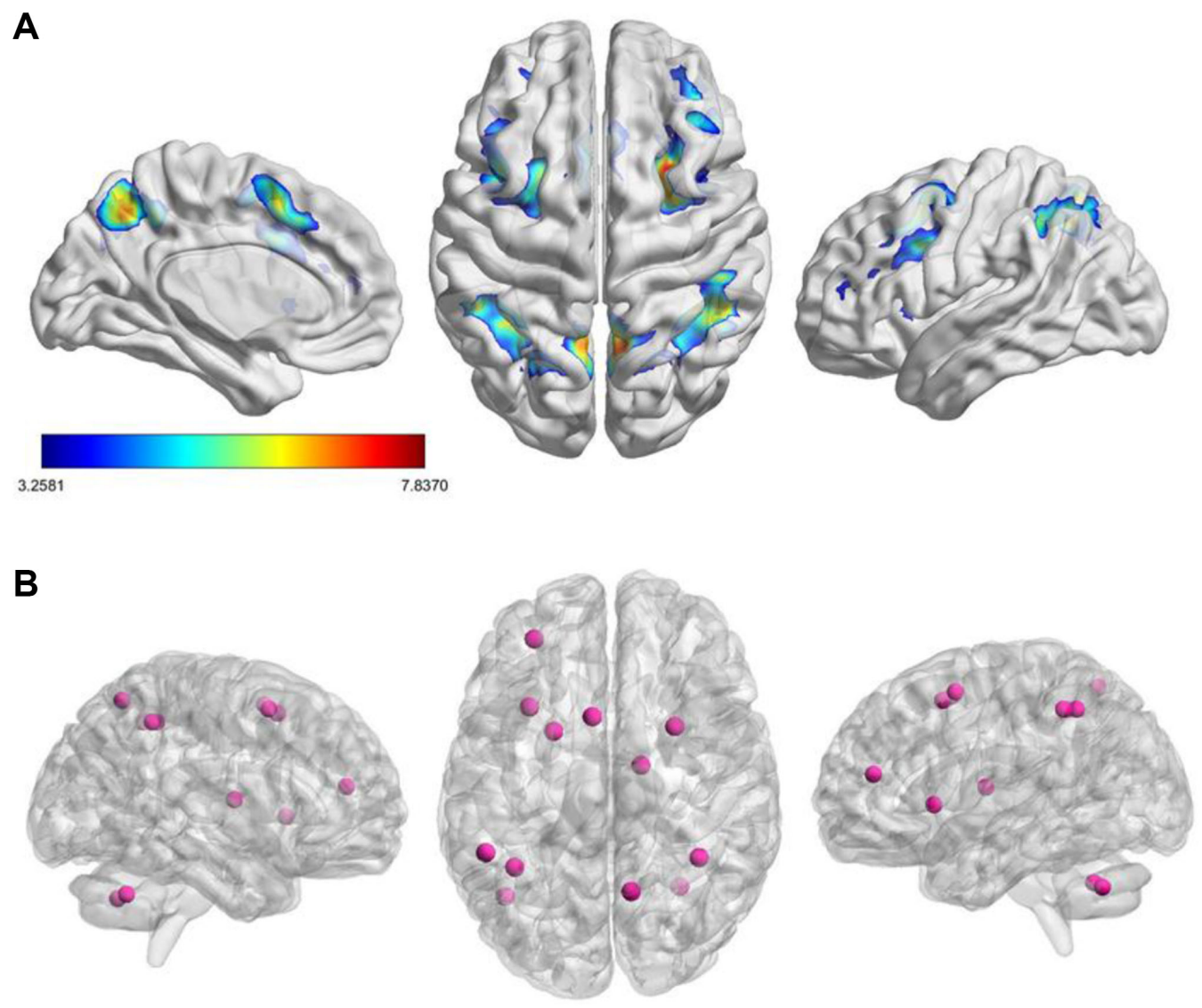

Supplementary Figure 2 . Working memory network identified in the $\mathbf{2 b a c k - 0 b a c k}$ contrast across all participants, during the n-back fMRI task. (A) Activation map, (B) 12 nodes reflecting the activation peaks (coordinates are provided in Supplementary Table 6).
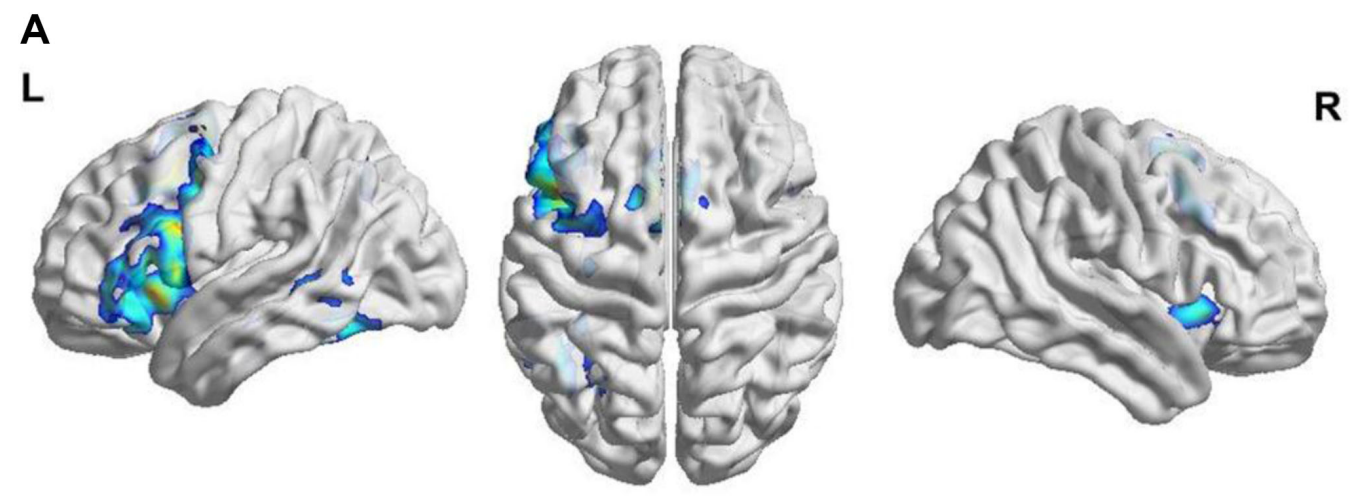

B
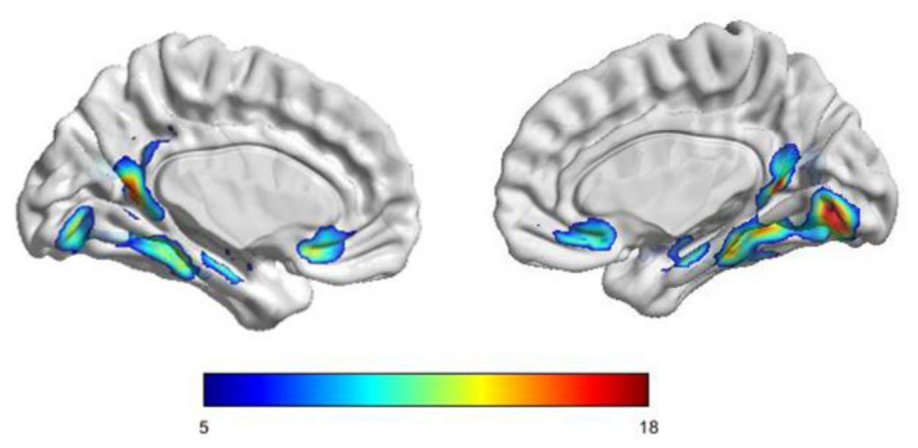

Supplementary Figure 3. Activation Maps for the Verb Generation (A) and Scene Encoding Memory Task (B). 


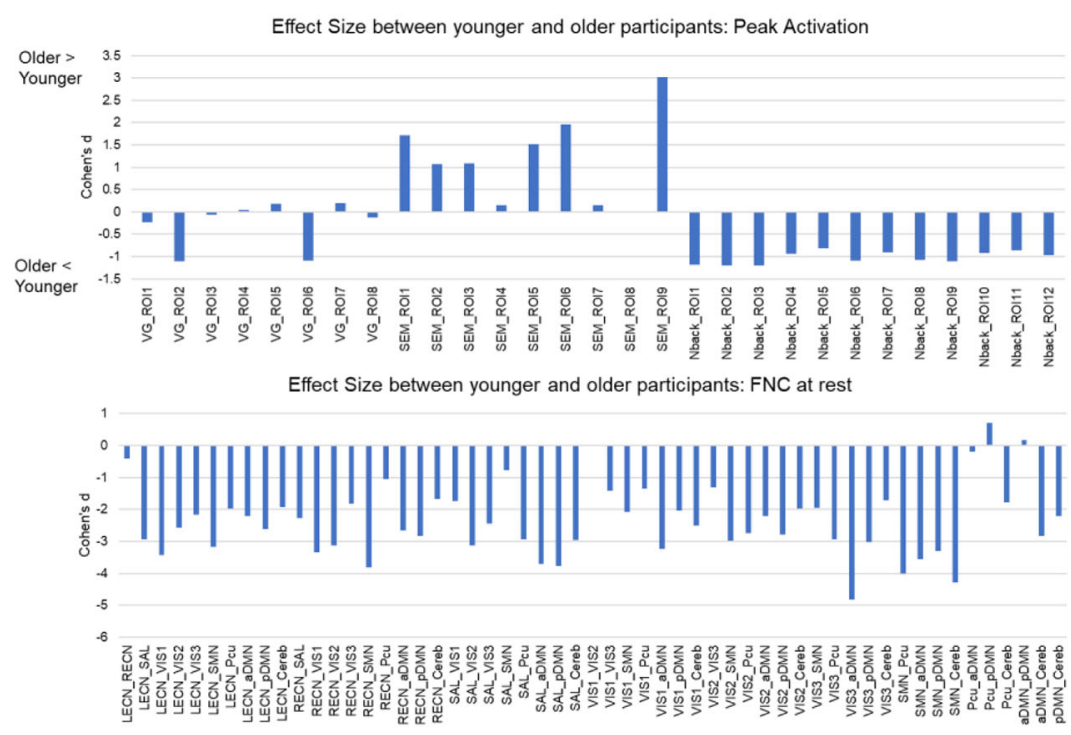

Effect Size between younger and older participants: FNC during VG
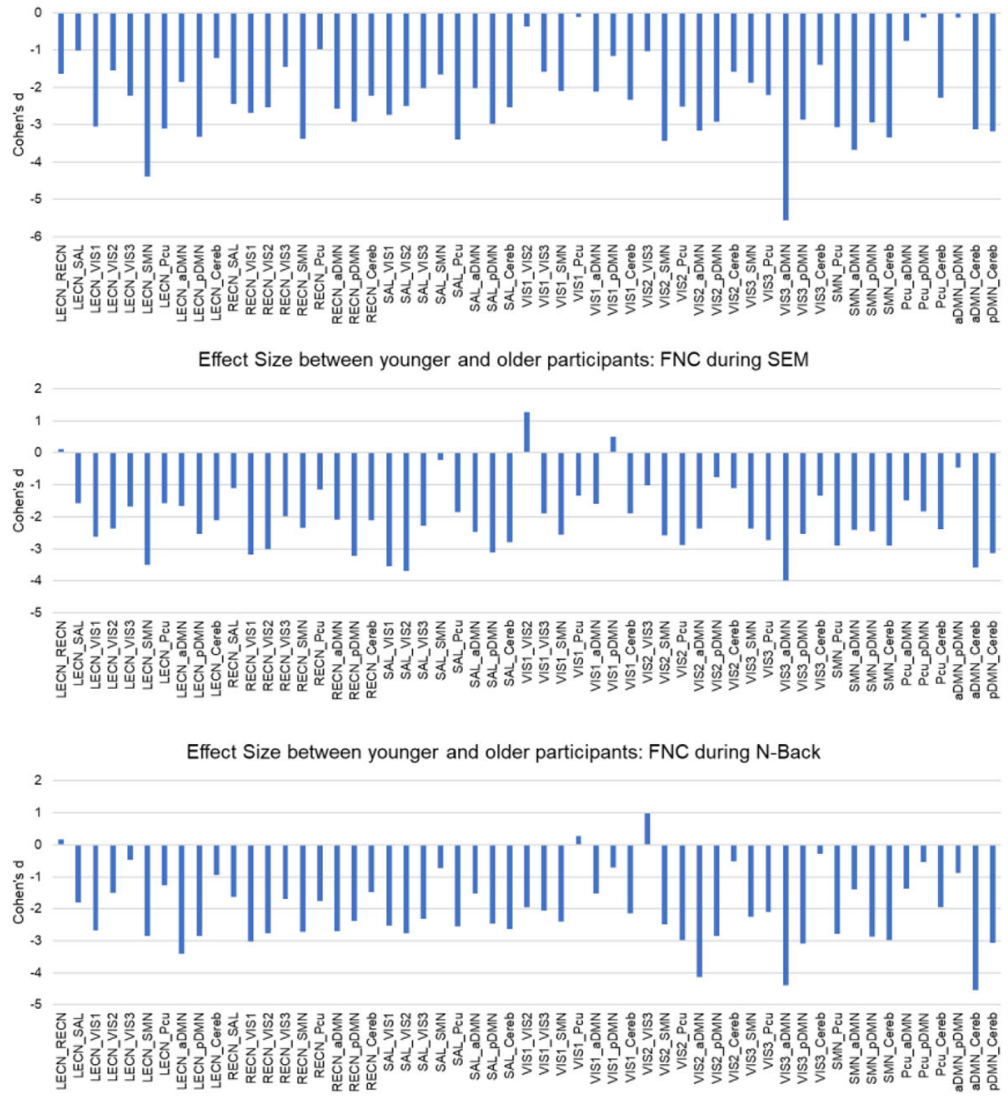

Supplementary Figure 4. Effect size between younger and older participants for each functional variable. A positive score reflects higher activation/connectivity in the older than in the younger participants. 


\section{Supplementary Tables}

Supplementary Table 1. Canonical weights of the non-imaging variables to the sMRI variate.

\begin{tabular}{|c|c|c|c|}
\hline Non-imaging Variable & $\begin{array}{l}\text { All participants: } \\
\text { sCCA Weights }\end{array}$ & $\begin{array}{c}\text { Younger participants only: } \\
\text { sCCA Weights }\end{array}$ & $\begin{array}{c}\text { Older participants only: } \\
\text { sCCA Weights }\end{array}$ \\
\hline Semantic fluency (animal_name) & $r=0.53$ & $\mathrm{r}=0.34$ & $\mathrm{r}=0.44$ \\
\hline Perceived stress (emo_psff_t) & $r=0.51$ & $\mathrm{r}=0.11$ & $\mathrm{r}=0.22$ \\
\hline Anger-Hostility (emo_ahff_t) & $r=0.44$ & $\mathrm{r}=0.003$ & $\mathbf{r}=\mathbf{0 . 3 2}$ \\
\hline Sadness (emo_sff_t) & $r=0.43$ & $\mathrm{r}=0.23$ & $\mathrm{r}=0.23$ \\
\hline Block_Design & $r=0.43$ & $\mathrm{r}=-0.29$ & $\mathbf{r}=\mathbf{0 . 3 9}$ \\
\hline 2_back_ACC & $r=0.42$ & $\mathrm{r}=-0.12$ & $\mathrm{r}=0.03$ \\
\hline Apathy (emo_aff_t) & $r=0.36$ & $\mathbf{r}=\mathbf{0 . 4 3}$ & $\mathrm{r}=0.21$ \\
\hline Loneliness (emo_lff_t) & $\mathbf{r}=\mathbf{0 . 3 5}$ & $\mathrm{r}=0.08$ & $\mathrm{r}=0.22$ \\
\hline Perceived hostility (emo_phff_t) & $\mathbf{r}=\mathbf{0 . 3 4}$ & $\mathrm{r}=0.05$ & $\mathrm{r}=0.13$ \\
\hline 0_back_ACC & $\mathbf{r}=\mathbf{0 . 3 4}$ & $\mathrm{r}=-0.08$ & $\mathrm{r}=0.07$ \\
\hline Matrix_Reasoning & $\mathrm{r}=0.33$ & $\mathrm{r}=-0.11$ & $\mathrm{r}=0.1$ \\
\hline Apathy (emo_aff_t) & $\mathrm{r}=0.32$ & $\mathrm{r}=0.06$ & $\mathrm{r}=0.18$ \\
\hline Fear-Affect (emo_faff_t) & $\mathrm{r}=0.31$ & $\mathrm{r}=-0.06$ & $\mathbf{r}=\mathbf{0 . 2 8}$ \\
\hline SEM_Scramble_ACC & $\mathrm{r}=0.3$ & $\mathrm{r}=-0.28$ & $\mathrm{r}=0.2$ \\
\hline Crystalized intelligence ( $\left.\operatorname{cog}_{-} \mathrm{cc} \_t\right)$ & $\mathrm{r}=0.25$ & $\mathbf{r}=\mathbf{0 . 3 7}$ & $\mathrm{r}=-0.19$ \\
\hline Physical activity & $\mathrm{r}=0.21$ & $\mathrm{r}=0.13$ & $\mathrm{r}=-0.27$ \\
\hline Testosterone & $\mathrm{r}=0.21$ & $\mathrm{r}=-0.01$ & $\mathrm{r}=0.03$ \\
\hline Perceived rejection (emo_prff_t) & $\mathrm{r}=0.2$ & $\mathrm{r}=0.36$ & $\mathrm{r}=-0.18$ \\
\hline Oral reading recognition ( $\operatorname{cog} \_$orr_t ) & $\mathrm{r}=0.2$ & $\mathrm{r}=0.03$ & $\mathrm{r}=-0.04$ \\
\hline Verbal fluency (total_FAS) & $\mathrm{r}=0.2$ & $\mathrm{r}=-0.1$ & $\mathrm{r}=0.22$ \\
\hline Weekend night sleep & $\mathrm{r}=0.18$ & $\mathrm{r}=0.07$ & $\mathrm{r}=0.15$ \\
\hline Sex & $\mathrm{r}=0.17$ & $\mathrm{r}=0.34$ & $\mathrm{r}=-0.02$ \\
\hline MMSE_Total & $\mathrm{r}=0.16$ & $\mathrm{r}=-0.14$ & $\mathrm{r}=0.21$ \\
\hline Fear-Somatic arousal (emo_fsaff_t) & $\mathrm{r}=0.15$ & $\mathrm{r}=-0.19$ & $\mathrm{r}=-0.21$ \\
\hline Similarities & $\mathrm{r}=0.09$ & $\mathrm{r}=-0.28$ & $\mathrm{r}=0.2$ \\
\hline Vocabulary & $\mathrm{r}=0.09$ & $\mathrm{r}=-0.17$ & $\mathrm{r}=0.17$ \\
\hline SEM_Scramble_RT & $\mathrm{r}=0.08$ & $\mathrm{r}=0.32$ & $\mathbf{r}=\mathbf{0 . 2 7}$ \\
\hline $\begin{array}{l}\text { Anger-Physical aggression } \\
\text { (emo_apaff_t) }\end{array}$ & $\mathrm{r}=0.06$ & $\mathrm{r}=-0.12$ & $\mathrm{r}=-0.23$ \\
\hline FSIQ & $\mathrm{r}=0.06$ & $\mathrm{r}=-0.31$ & $\mathrm{r}=0.21$ \\
\hline Picture sorting memory $\left(\operatorname{cog} \_p s m \_t\right)$ & $\mathrm{r}=0.06$ & $\mathrm{r}=-0.22$ & $\mathrm{r}=-0.15$ \\
\hline Estradiol & $\mathrm{r}=0.04$ & $\mathrm{r}=-0.01$ & $\mathrm{r}=-0.2$ \\
\hline Weeknight sleep & $\mathrm{r}=0.04$ & $\mathrm{r}=0.01$ & $\mathrm{r}=0.05$ \\
\hline Number of alcoholic drinks & $\mathrm{r}=0.01$ & $\mathrm{r}=-0.34$ & $\mathrm{r}=0.06$ \\
\hline Picture vocabulary (cog_pv_t) & $\mathrm{r}=0.002$ & $\mathrm{r}=0.21$ & $\mathrm{r}=-0.23$ \\
\hline BMI & $\mathrm{r}=-0.01$ & $\mathbf{r}=\mathbf{0 . 4 7}$ & $\mathrm{r}=0.15$ \\
\hline $\begin{array}{l}\text { Dimensional card change sort } \\
\left(\operatorname{cog} \_d c c s \_t\right)\end{array}$ & $\mathrm{r}=-0.06$ & $\mathrm{r}=-0.26$ & $\mathrm{r}=-0.04$ \\
\hline SEM_Scene_RT & $\mathrm{r}=-0.07$ & $\mathbf{r}=\mathbf{0 . 3 8}$ & $\mathrm{r}=0.16$ \\
\hline Pattern comparison (cog_pcps_t) & $\mathrm{r}=-0.08$ & $\mathrm{r}=-0.38$ & $\mathrm{r}=-0.06$ \\
\hline SEM_Scene_ACC & $\mathrm{r}=-0.09$ & $\mathrm{r}=-0.36$ & $\mathrm{r}=0.12$ \\
\hline Fluid intelligence (cog_fc_t) & $\mathrm{r}=-0.12$ & $r=-0.49$ & $\mathrm{r}=-0.21$ \\
\hline $\begin{array}{l}\text { Flanker inhibitory control and } \\
\text { attention (cog_fica_t) }\end{array}$ & $r=-0.15$ & $\mathrm{r}=-0.26$ & $\mathrm{r}=-0.25$ \\
\hline EAG & $\mathrm{r}=-0.16$ & $\mathrm{r}=0.25$ & $\mathrm{r}=0.05$ \\
\hline
\end{tabular}




\begin{tabular}{|c|c|c|c|}
\hline HBA1C & $\mathrm{r}=-0.16$ & $\mathrm{r}=0.25$ & $\mathrm{r}=0.04$ \\
\hline Friendship (emo_fff_t) & $\mathrm{r}=-0.21$ & $\mathbf{r}=-0.5$ & $\mathbf{r}=-\mathbf{0 . 3 7}$ \\
\hline $\begin{array}{l}\text { List sorting working memory } \\
\left(\operatorname{cog} \_l s w m \_t\right)\end{array}$ & $\mathrm{r}=-0.26$ & $\mathbf{r}=-\mathbf{0 . 5 1}$ & $\mathrm{r}=-0.12$ \\
\hline High blood pressure & $\mathrm{r}=-0.31$ & $\mathrm{r}=-0.11$ & $\mathbf{r}=-\mathbf{0 . 3 7}$ \\
\hline 0_back_RT & $\mathrm{r}=-0.32$ & $\mathrm{r}=0.22$ & $\mathrm{r}=0.08$ \\
\hline 2_back_RT & $\mathbf{r}=-\mathbf{0 . 3 4}$ & $\mathrm{r}=0.19$ & $\mathrm{r}=-0.17$ \\
\hline Instrumental support (emo_isff_t) & $\mathbf{r}=-\mathbf{0 . 3 6}$ & $\mathrm{r}=-0.38$ & $\mathrm{r}=-0.03$ \\
\hline Pegboard_LH & $\mathbf{r}=-\mathbf{0 . 3 7}$ & $\mathrm{r}=-0.03$ & $\mathrm{r}=-0.09$ \\
\hline Social satisfaction (emo_sss_t) & $\mathbf{r}=-\mathbf{0 . 4}$ & $\mathrm{r}=-0.45$ & $\mathrm{r}=-0.2$ \\
\hline Self-efficacy (emo_secat_t) & $\mathbf{r}=-\mathbf{0 . 4 2}$ & $\mathrm{r}=-0.31$ & $\mathrm{r}=-0.23$ \\
\hline Meaning and Purpose (emo_mpff_t $)$ & $\mathbf{r}=-\mathbf{0 . 4 2}$ & $\mathrm{r}=-0.19$ & $\mathbf{r}=-\mathbf{0 . 3 7}$ \\
\hline General life satisfaction (emo_glsff_t) & $\mathbf{r}=-\mathbf{0 . 5 5}$ & $\mathrm{r}=-0.26$ & $\mathbf{r}=-\mathbf{0 . 5 1}$ \\
\hline Age & $r=-0.74$ & $\mathrm{r}=0.15$ & $r=-0.57$ \\
\hline
\end{tabular}

Variables are described in Supplementary Table 5. Top contributions shown in the main text are highlighted in bold.

Supplementary Table 2. Canonical weights of the sMRI variables to the non-imaging variate.

\begin{tabular}{|c|c|c|c|}
\hline Region & $\begin{array}{l}\text { All Subjects: } \\
\text { sCCA Weights }\end{array}$ & $\begin{array}{l}\text { Younger Participants Only: } \\
\text { sCCA Weights }\end{array}$ & $\begin{array}{c}\text { Older Participants Only: } \\
\text { sCCA Weights }\end{array}$ \\
\hline Right Superior Frontal Thickness & $r=0.74$ & $r=0.48$ & $r=0.51$ \\
\hline Left Superior Frontal Thickness & $\mathbf{r}=\mathbf{0 . 7 2}$ & $\mathbf{r}=\mathbf{0 . 3 9}$ & $r=0.48$ \\
\hline Left Pars Opercularis Thickness & $\mathbf{r}=0.7$ & $\mathrm{r}=0.21$ & $\mathbf{r}=\mathbf{0 . 5 1}$ \\
\hline Right Pars Opercularis Thickness & $\mathbf{r}=\mathbf{0 . 6 4}$ & $\mathrm{r}=0.17$ & $\mathrm{r}=0.4$ \\
\hline Right Insula Thickness & $r=0.63$ & $\mathrm{r}=0.07$ & $\mathrm{r}=0.32$ \\
\hline Left Precentral Thickness & $\mathbf{r}=\mathbf{0 . 6 2}$ & $\mathrm{r}=-0.05$ & $r=0.46$ \\
\hline Left Pars Triangularis Thickness & $\mathbf{r}=\mathbf{0 . 6}$ & $\mathrm{r}=0.19$ & $\mathrm{r}=0.41$ \\
\hline Left Superior Temporal Thickness & $\mathbf{r}=\mathbf{0 . 5 8}$ & $\mathrm{r}=0.19$ & $\mathrm{r}=0.31$ \\
\hline Right Paracentral Thickness & $\mathbf{r}=\mathbf{0 . 5 8}$ & $\mathrm{r}=0.01$ & $\mathbf{r}=\mathbf{0 . 5 1}$ \\
\hline Right Precuneus Thickness & $\mathbf{r}=\mathbf{0 . 5 4}$ & $\mathbf{r}=\mathbf{0 . 3 1}$ & $\mathrm{r}=0.38$ \\
\hline Right Precentral Thickness & $\mathbf{r}=\mathbf{0 . 5 2}$ & $\mathbf{r}=-\mathbf{0 . 2 5}$ & $\mathbf{r}=\mathbf{0 . 4 5}$ \\
\hline Right Caudal Middle Frontal Thickness & $\mathbf{r}=\mathbf{0 . 5 2}$ & $\mathrm{r}=0.18$ & $\mathrm{r}=0.26$ \\
\hline Right Superior Parietal Thickness & $\mathbf{r}=\mathbf{0 . 5 2}$ & $\mathrm{r}=0.01$ & $\mathbf{r}=\mathbf{0 . 5 3}$ \\
\hline Left Inferior Parietal Thickness & $\mathrm{r}=0.5$ & $\mathrm{r}=0.35$ & $\mathrm{r}=0.34$ \\
\hline Right Supramarginal Thickness & $r=0.49$ & $r=0.07$ & $\mathrm{r}=0.28$ \\
\hline $\begin{array}{l}\text { Left Rostral Anterior Cingulate } \\
\text { Thickness }\end{array}$ & $\mathrm{r}=0.49$ & $\mathrm{r}=0.22$ & $\mathrm{r}=0.13$ \\
\hline Left Insula Thickness & $\mathrm{r}=0.49$ & $\mathrm{r}=0.09$ & $\mathrm{r}=0.06$ \\
\hline Left Postcentral Thickness & $\mathrm{r}=0.47$ & $\mathrm{r}=-0.07$ & $\mathbf{r}=\mathbf{0 . 6 2}$ \\
\hline
\end{tabular}


Left Supramarginal Thickness

Left Accumbens Volume

Right Putamen Volume

Left Middle Temporal Thickness

Left Pallidum Volume

Left Superior Parietal Thickness

Left Amygdala Volume

Right Middle Temporal Thickness

Left Precuneus Thickness

Right Rostral Middle Frontal Thickness

Left Putamen Volume

Right Pallidum Volume

Left Rostral Middle Frontal Thickness

Left Posterior Cingulate Thickness

Right Thalamus Volume

Right Postcentral Thickness

Right Posterior Cingulate Thickness

Right Lateral Orbitofrontal Thickness

Right Rostral Anterior Cingulate

Thickness

Right Accumbens Volume

Right Amygdala Volume

Left Lateral Orbitofrontal Thickness

Right Pars Orbitalis Thickness

Left Isthmus Cingulate Thickness

Left Paracentral Thickness

Left Caudal Anterior Cingulate

Thickness

Left Caudate Volume

Left Hippocampus Volume

Right Bankssts Thickness

Right Cerebellum Cortex Volume

Left Pars Orbitalis Thickness

Right Hippocampus Volume

Left Cerebellum Cortex Volume

Left Parahippocampal Thickness

Left Medial Orbitofrontal Thickness

Right Inferior Temporal Thickness

Left Inferior Temporal Thickness

Left Frontal Pole Thickness

Right Entorhinal Thickness

Left Lateral Occipital Thickness

Right Frontal Pole Thickness

Left Transverse Temporal Thickness

Right Parahippocampal Thickness

Right Caudate Volume

Right Temporal Pole Thickness

Left Fusiform Thickness

Right Cuneus Thickness $\mathrm{r}=0.46$

$\mathrm{r}=0.45$

$\mathrm{r}=0.44$

$\mathrm{r}=0.44$

$\mathrm{r}=0.43$

$\mathrm{r}=0.42$

$\mathrm{r}=0.42$

$\mathrm{r}=0.42$

$\mathrm{r}=0.41$

$\mathrm{r}=0.41$

$\mathrm{r}=0.39$

$\mathrm{r}=0.38$

$\mathrm{r}=0.38$

$\mathrm{r}=0.36$

$\mathrm{r}=0.33$

$\mathrm{r}=0.33$

$\mathrm{r}=0.33$

$\mathrm{r}=0.32$

$\mathrm{r}=0.32$

$\mathrm{r}=0.31$

$\mathrm{r}=0.31$

$\mathrm{r}=0.31$

$\mathrm{r}=0.3$

$\mathrm{r}=0.3$

$\mathrm{r}=0.3$

$\mathrm{r}=0.29$

$\mathrm{r}=0.28$

$\mathrm{r}=0.28$

$\mathrm{r}=0.28$

$\mathrm{r}=0.27$

$\mathrm{r}=0.27$

$\mathrm{r}=0.27$

$\mathrm{r}=0.25$

$\mathrm{r}=0.24$

$\mathrm{r}=0.24$

$\mathrm{r}=0.24$

$\mathrm{r}=0.21$

$\mathrm{r}=0.21$

$\mathrm{r}=0.2$

$\mathrm{r}=0.19$

$\mathrm{r}=0.19$

$\mathrm{r}=0.18$

$\mathrm{r}=0.15$

$\mathrm{r}=0.15$

$\mathrm{r}=0.15$

$\mathrm{r}=0.13$

$\mathrm{r}=0.12$ $\mathrm{r}=0.1$

$\mathrm{r}=0.02$

$\mathrm{r}=0.09$

$\mathrm{r}=-0.03$

$\mathbf{r}=\mathbf{- 0 . 1 9}$

$\mathrm{r}=0.28$

$\mathrm{r}=-0.03$

$\mathbf{r}=\mathbf{- 0 . 2 1}$

$\mathrm{r}=0.19$

$\mathbf{r}=\mathbf{0 . 3 3}$

$\mathrm{r}=0.01$

$\mathrm{r}=-0.1$

$\mathrm{r}=0.13$

$\mathrm{r}=-0.06$

$\mathrm{r}=-0.1$

$\mathrm{r}=-0.17$

$\mathrm{r}=0.02$

$\mathrm{r}=0.27$

$\mathrm{r}=0.21$

$\mathrm{r}=0.09$

$\mathrm{r}=0.02$

$\mathrm{r}=0.24$

$\mathrm{r}=0.06$

$\mathbf{r}=\mathbf{- 0 . 1 7}$

$\mathrm{r}=0.12$

$\mathrm{r}=0.27$

$\mathrm{r}=-0.01$

$\mathrm{r}=-0.05$

$\mathrm{r}=0.11$

$\mathrm{r}=0.13$

$r=-0.28$

$r=-0.12$

$\mathrm{r}=0.11$

$\mathrm{r}=-0.05$

$\mathrm{r}=0.09$

$\mathrm{r}=-0.1$

$\mathrm{r}=-0.05$

$\mathrm{r}=0.02$

$\mathrm{r}=0.12$

$\mathrm{r}=-0.01$

$\mathbf{r}=\mathbf{0 . 5 3}$

$\mathrm{r}=0.07$

$\mathrm{r}=0.04$

$\mathrm{r}=-0.03$

$\mathrm{r}=-0.03$

$\mathrm{r}=0.01$

$\mathrm{r}=0.03$ $\mathrm{r}=0.16$

$\mathrm{r}=0.22$

$\mathrm{r}=0.05$

$\mathrm{r}=0.17$

$\mathrm{r}=0.39$

$\mathbf{r}=\mathbf{0 . 4 7}$

$\mathrm{r}=0.34$

$\mathrm{r}=0.01$

$\mathrm{r}=0.4$

$\mathrm{r}=0.17$

$\mathrm{r}=0.21$

$\mathbf{r}=\mathbf{0 . 4 5}$

$\mathrm{r}=0.29$

$\mathrm{r}=-0.04$

$\mathrm{r}=0.12$

$\mathbf{r}=\mathbf{0 . 4 4}$

$\mathrm{r}=0.04$

$\mathrm{r}=-0.06$

$\mathrm{r}=0.08$

$\mathrm{r}=0.09$

$\mathrm{r}=0.2$

$r=-0.04$

$\mathrm{r}=0.03$

$\mathrm{r}=0.11$

$\mathrm{r}=0.29$

$\mathrm{r}=0.22$

$\mathbf{r}=\mathbf{- 0 . 1 7}$

$\mathrm{r}=0.17$

$\mathrm{r}=0.28$

$\mathrm{r}=0.01$

$\mathrm{r}=0.17$

$\mathrm{r}=0.27$

$\mathrm{r}=-0.02$

$\mathrm{r}=0.26$

$\mathrm{r}=-0.02$

$\mathrm{r}=0.06$

$\mathrm{r}=0.03$

$\mathrm{r}=0.36$

$\mathbf{r}=\mathbf{- 0 . 1}$

$\mathrm{r}=0.35$

$\mathrm{r}=-0.04$

$\mathrm{r}=0.07$

$\mathrm{r}=0.08$

$\mathbf{r}=-\mathbf{0 . 2 1}$

$\mathrm{r}=0.16$

$\mathrm{r}=0.02$

$\mathrm{r}=0.11$ 


\begin{tabular}{lccc} 
Right Transverse Temporal Thickness & $\mathrm{r}=0.12$ & $\mathrm{r}=-0.08$ & $\mathrm{r}=0.1$ \\
Right Isthmus Cingulate Thickness & $\mathrm{r}=0.11$ & $\mathrm{r}=-0.05$ & $\mathrm{r}=0.22$ \\
Right Lateral Occipital Thickness & $\mathrm{r}=0.1$ & $\mathbf{r}=-\mathbf{0 . 1 3}$ & $\mathrm{r}=0.19$ \\
Right Caudal Anterior Cingulate & $\mathrm{r}=0.08$ & $\mathbf{r}=\mathbf{0 . 3 4}$ & $\mathbf{r}=\mathbf{- 0 . 1 8}$ \\
Thickness & $\mathrm{r}=0.07$ & $\mathrm{r}=0.13$ & $\mathrm{r}=-0.03$ \\
Left Pericalcarine Thickness & $\mathrm{r}=0.04$ & $\mathbf{r}=-\mathbf{0 . 1 3}$ & $\mathrm{r}=0.15$ \\
Left Entorhinal Thickness & $\mathrm{r}=0.04$ & $\mathrm{r}=0.06$ & $\mathrm{r}=0$ \\
Left Temporal Pole Thickness & $\mathrm{r}=0.01$ & $\mathrm{r}=-0.1$ & $\mathrm{r}=0$ \\
Right Fusiform Thickness & $\mathrm{r}=-0.03$ & $\mathbf{r}=-\mathbf{0 . 3 4}$ & $\mathrm{r}=-0.07$ \\
Right Lingual Thickness & $\mathrm{r}=-0.04$ & $\mathrm{r}=0.03$ & $\mathrm{r}=0.01$ \\
Left Cuneus Thickness & $\mathrm{r}=-0.04$ & $\mathrm{r}=0.16$ & $\mathrm{r}=-0.04$ \\
Right Pericalcarine Thickness & $\mathrm{r}=-0.08$ & $\mathrm{r}=0.03$ & $\mathbf{r}=-\mathbf{0 . 2 8}$ \\
Left Lingual Thickness & $\mathrm{r}=-0.13$ & $\mathrm{r}=0.16$ & $\mathbf{r}=-\mathbf{0 . 4 7}$ \\
Right Medial Orbitofrontal Thickness & $\mathrm{r}=-0.58$ & $\mathrm{r}=-0.08$ & $\mathbf{r}=-\mathbf{0 . 3 7}$ \\
Right Lateral Ventricle Volume & $\mathrm{r}=-0.6$ & $\mathrm{r}=-0.04$ & $\mathbf{r}=\mathbf{- 0 . 4 8}$ \\
Left Lateral Ventricle Volume & & & \\
\hline
\end{tabular}

Top contributions shown in the main text are highlighted in bold.

Supplementary Table 3. Canonical weights of the non-imaging variables to the fMRI variate.

\begin{tabular}{|c|c|c|c|}
\hline Non-imaging Variable & $\begin{array}{l}\text { All participants: } \\
\text { sCCA Weights }\end{array}$ & $\begin{array}{c}\text { Younger participants only: } \\
\text { SCCA Weights }\end{array}$ & $\begin{array}{l}\text { Older participants only: } \\
\text { sCCA Weights }\end{array}$ \\
\hline 2_back_ACC & $\mathrm{r}=\mathbf{0 . 6 8}$ & $\mathrm{r}=0.08$ & $\mathrm{r}=0.5$ \\
\hline Perceived stress (emo_psff_t $)$ & $\mathrm{r}=\mathbf{0 . 6 8}$ & $\mathrm{r}=\mathbf{0 . 4 2}$ & $r=0.48$ \\
\hline Matrix_Reasoning & $\mathbf{r}=\mathbf{0 . 5 6}$ & $\mathrm{r}=-0.05$ & $\mathbf{r}=\mathbf{0 . 5 9}$ \\
\hline Block_Design & $\mathrm{r}=\mathbf{0 . 5 6}$ & $\mathrm{r}=0.04$ & $r=0.26$ \\
\hline Anger-Hostility (emo_ahff_t) & $\mathbf{r}=\mathbf{0 . 5 5}$ & $\mathrm{r}=0.23$ & $r=0.43$ \\
\hline Loneliness (emo_lff_t) & $\mathrm{r}=\mathbf{0 . 4 9}$ & $\mathbf{r}=\mathbf{0 . 3 4}$ & $\mathrm{r}=\mathbf{0 . 4 8}$ \\
\hline Perceived hostility (emo_phff_t) & $\mathrm{r}=\mathbf{0 . 4 6}$ & $\mathrm{r}=0.23$ & $\mathrm{r}=0.3$ \\
\hline Sadness (emo_sff_t) & $\mathrm{r}=\mathbf{0 . 4 5}$ & $\mathrm{r}=0.26$ & $\mathrm{r}=0.23$ \\
\hline Perceived rejection (emo_prff_t) & $\mathrm{r}=\mathbf{0 . 4 3}$ & $\mathrm{r}=0.19$ & $r=0.69$ \\
\hline Fear-Somatic arousal (emo_fsaff_t) & $\mathrm{r}=\mathbf{0 . 4 2}$ & $\mathrm{r}=0.26$ & $r=0.18$ \\
\hline Anger-Affect (emo_aff_t) & $\mathrm{r}=0.4$ & $\mathbf{r}=\mathbf{0 . 3 1}$ & $\mathrm{r}=0.25$ \\
\hline Fear-Affect (emo_faff_t $)$ & $\mathrm{r}=0.4$ & $\mathrm{r}=0.26$ & $\mathrm{r}=0.22$ \\
\hline Physical activity & $\mathrm{r}=0.39$ & $\mathrm{r}=-0.14$ & $\mathrm{r}=0.17$ \\
\hline 0_back_ACC & $\mathrm{r}=0.39$ & $\mathrm{r}=0.004$ & $\mathrm{r}=-0.02$ \\
\hline SEM_Scramble_ACC & $\mathrm{r}=0.36$ & $\mathrm{r}=-0.16$ & $\mathrm{r}=0.17$ \\
\hline Oral reading recognition ( $\operatorname{cog}_{-}$orr_t $\mathrm{t}$ ) & $\mathrm{r}=0.32$ & $\mathbf{r}=\mathbf{0 . 4}$ & $\mathrm{r}=0.002$ \\
\hline Picture sorting memory (cog_psm_t) & $\mathrm{r}=0.31$ & $\mathrm{r}=0.11$ & $\mathrm{r}=0.26$ \\
\hline Testosterone & $\mathrm{r}=0.3$ & $\mathrm{r}=0.25$ & $r=0.02$ \\
\hline Apathy (emo_aff_t) & $\mathrm{r}=0.3$ & $\mathrm{r}=0.15$ & $r=0.37$ \\
\hline $\begin{array}{l}\text { Anger-Physical aggression } \\
\text { (emo_apaff_t) }\end{array}$ & $\mathrm{r}=0.28$ & $\mathrm{r}=0.22$ & $\mathrm{r}=0.14$ \\
\hline Semantic fluency (animal_name) & $\mathrm{r}=0.25$ & $\mathrm{r}=0.15$ & $\mathrm{r}=-0.32$ \\
\hline Estradiol & $\mathrm{r}=0.24$ & $r=0.46$ & $\mathrm{r}=0.09$ \\
\hline Crystalized intelligence $\left(\operatorname{cog} \_c c \_t\right)$ & $\mathrm{r}=0.23$ & $\mathrm{r}=0.18$ & $\mathrm{r}=0.12$ \\
\hline FSIQ & $\mathrm{r}=0.2$ & $\mathrm{r}=-0.06$ & $r=0.36$ \\
\hline Vocabulary & $\mathrm{r}=0.19$ & $\mathrm{r}=0.13$ & $r=0.24$ \\
\hline Pattern comparison (cog_pcps_t ) & $\mathrm{r}=0.18$ & $\mathrm{r}=0.23$ & $\mathrm{r}=0.22$ \\
\hline
\end{tabular}




\begin{tabular}{|c|c|c|c|}
\hline Fluid intelligence (cog_fc_t) & $\mathrm{r}=0.14$ & $\mathrm{r}=0.09$ & $\mathrm{r}=-0.06$ \\
\hline Similarities & $\mathrm{r}=0.13$ & $\mathrm{r}=-0.01$ & $\mathrm{r}=-0.01$ \\
\hline MMSE_Total & $\mathrm{r}=0.12$ & $\mathrm{r}=0.14$ & $\mathrm{r}=-0.13$ \\
\hline Verbal fluency (total_FAS) & $\mathrm{r}=0.11$ & $\mathrm{r}=0.01$ & $\mathrm{r}=-0.31$ \\
\hline Number of alcoholic drinks & $\mathrm{r}=0.09$ & $\mathrm{r}=0.1$ & $\mathrm{r}=-0.01$ \\
\hline Weekend night sleep & $\mathrm{r}=0.09$ & $\mathbf{r}=-\mathbf{0 . 3 1}$ & $\mathrm{r}=0.04$ \\
\hline Sex & $\mathrm{r}=0.07$ & $\mathrm{r}=0.07$ & $\mathrm{r}=-0.07$ \\
\hline $\begin{array}{l}\text { Flanker inhibitory control and } \\
\text { attention (cog_fica_t) }\end{array}$ & $\mathrm{r}=0.06$ & $r=0.26$ & $\mathrm{r}=-0.02$ \\
\hline Weeknight sleep & $\mathrm{r}=0.05$ & $\mathrm{r}=-0.22$ & $\mathrm{r}=0.25$ \\
\hline Picture vocabulary $\left(\operatorname{cog} \_p v \_t\right)$ & $\mathrm{r}=0.01$ & $\mathrm{r}=-0.07$ & $\mathrm{r}=0.21$ \\
\hline SEM_Scramble_MeanRT & $\mathrm{r}=-0.02$ & $\mathrm{r}=0.17$ & $\mathrm{r}=0.31$ \\
\hline SEM_Scene_ACC & $\mathrm{r}=-0.03$ & $\mathrm{r}=-0.02$ & $\mathrm{r}=0.07$ \\
\hline $\begin{array}{l}\text { Dimensional card change sort } \\
\left(\operatorname{cog} \_d c c s \_t\right)\end{array}$ & $\mathrm{r}=-0.04$ & $\mathrm{r}=-0.15$ & $\mathrm{r}=-0.25$ \\
\hline Friendship (emo_fff_t) & $\mathrm{r}=-0.06$ & $\mathrm{r}=-0.28$ & $\mathrm{r}=-\mathbf{0 . 4 2}$ \\
\hline High blood pressure & $\mathrm{r}=-0.14$ & $\mathrm{r}=0.12$ & $\mathrm{r}=-0.01$ \\
\hline SEM_Scene_MeanRT & $\mathrm{r}=-0.24$ & $\mathrm{r}=0.01$ & $\mathrm{r}=0.14$ \\
\hline $\begin{array}{l}\text { List sorting working memory } \\
(\operatorname{cog} l s w m-t)\end{array}$ & $\mathrm{r}=-0.26$ & $\mathrm{r}=-0.2$ & $\mathbf{r}=-\mathbf{0 . 5 9}$ \\
\hline 2_back_MeanRT & $\mathrm{r}=-0.3$ & $\mathrm{r}=0.19$ & $\mathrm{r}=0.26$ \\
\hline BMI & $\mathrm{r}=-0.31$ & $\mathrm{r}=-0.12$ & $\mathrm{r}=-0.11$ \\
\hline Emotional support (emo_esff_t) & $\mathrm{r}=-0.33$ & $\mathrm{r}=-0.11$ & $\mathbf{r}=-\mathbf{0 . 3 9}$ \\
\hline $\begin{array}{l}\text { Domain life satisfaction } \\
\text { (emo_dslsff_raw) }\end{array}$ & $\mathrm{r}=-0.33$ & $\mathrm{r}=-0.04$ & $\mathbf{r}=-\mathbf{0 . 4 9}$ \\
\hline Positive affect (emo_paff_t) & $\mathrm{r}=-0.34$ & $\mathbf{r}=-\mathbf{0 . 4 1}$ & $\mathrm{r}=-0.34$ \\
\hline HBA1C & $\mathrm{r}=-0.35$ & $\mathrm{r}=0.0008$ & $\mathrm{r}=-0.16$ \\
\hline EAG & $\mathbf{r}=-\mathbf{0 . 3 5}$ & $\mathrm{r}=0.00001$ & $\mathrm{r}=-0.17$ \\
\hline Self-efficacy (emo_secat_t) & $\mathbf{r}=-0.4$ & $\mathrm{r}=-0.3$ & $\mathrm{r}=-0.2$ \\
\hline Instrumental support (emo_isff_t) & $\mathrm{r}=-\mathbf{0 . 4 2}$ & $\mathrm{r}=-0.15$ & $\mathrm{r}=-0.39$ \\
\hline Social satisfaction (emo_sss_t) & $r=-\mathbf{0 . 4 5}$ & $\mathrm{r}=-0.29$ & $r=-0.63$ \\
\hline Meaning and Purpose (emo_mpff_t) & $r=-0.46$ & $\mathbf{r}=-\mathbf{0 . 4}$ & $\mathrm{r}=-0.38$ \\
\hline 0_back_MeanRT & $r=-0.46$ & $\mathrm{r}=0.05$ & $\mathrm{r}=0.02$ \\
\hline General life satisfaction (emo_glsff_t) & $\mathrm{r}=-\mathbf{0 . 4 7}$ & $\mathrm{r}=-0.07$ & $\mathrm{r}=-0.38$ \\
\hline Pegboard_LH & $\mathbf{r}=-0.5$ & $\mathbf{r}=-\mathbf{0 . 3 7}$ & $\mathrm{r}=-0.2$ \\
\hline Pegboard_RH & $r=-0.6$ & $\mathrm{r}=-0.06$ & $\mathrm{r}=-0.33$ \\
\hline Age & $\mathbf{r}=-\mathbf{0 . 8 9}$ & $\mathbf{r}=-0.5$ & $\mathrm{r}=-0.3$ \\
\hline
\end{tabular}

Variables are described in Supplementary Table 5. Top contributions shown in the main text are highlighted in bold.

Supplementary Table 4. Demographic and cognitive information for each study group.

\begin{tabular}{lccc}
\hline Variables & $\begin{array}{c}\text { Younger Participants } \\
(\boldsymbol{n}=\mathbf{2 7})\end{array}$ & $\begin{array}{c}\text { Older Participants } \\
(\boldsymbol{n}=\mathbf{2 6})\end{array}$ & $\begin{array}{c}\boldsymbol{P} \text {-value } \\
\text { Sex (Females, } \boldsymbol{n}[\mathbf{\%}])\end{array}$ \\
Age (years) & $14[51.9 \%]$ & $17[65 \%]$ & 0.5160 \\
Body Mass Index (BMI) & $25.54(3.42)$ & $61.85(4.37)$ & 0.0000 \\
HBA1C & $24.34(3.67)$ & $26.63(3.79)$ & 0.0300 \\
EAG & $5.26(0.28)$ & $5.48(0.33)$ & 0.0130 \\
Estradiol & $104.20(8.00)$ & $110.5(9.36)$ & 0.0130 \\
Testosterone & $1.42(0.91)$ & $1.18(0.7)$ & 0.2810 \\
& $126.477(87.55)$ & $84.16(55.65)$ & 0.0420
\end{tabular}


High Blood Pressure (n [\%])

Number of Alcoholic Drinks

Physical Activity

Weeknight Sleep

Weekend Night Sleep

MMSE_total

Block_design

Vocabulary

Matrix_reasoning

Similarities

FSIQ

Verbal fluency (Total_FAS)

Semantic fluency (Animal_name)

(cog_orr_t)

cog_pv_t

cog_lswm_t

cog_pcps_t

cog_psm_t

cog_fica_t

cog_dces_t

$\operatorname{cog}$ fe_t

cog_cc_t

pegboard_rh

pegboard_lh

emo_ahff_t

emo_aaff_t

emo_apaff_t

emo_phff_t

emo Iff $t$

emo_prff_t

emo_secat_t

emo_psff_t

emo_faff_t

emo_fsaff_t

emo_glsff_t

emo_dslsff_raw

emo_mpff_t

emo_paff_t

emo_sff_t

emo_aff_t

emo_esff_t

emo_fff_t

emo_isff_t

emo_sss_t

SEM_Scene_MeanRT (ms)

SEM_Scene_ACC

SEM_Scramble_MeanRT (ms)

SEM_Scramble_ACC

$$
\begin{gathered}
2[7.4 \%] \\
4.07(1.73) \\
4.33(0.76) \\
7.96(1.15) \\
8.43(1.22) \\
28.96(1.43) \\
53.89(10.17) \\
41.63(3.66) \\
24.11(2.15) \\
35.93(3.63) \\
114.15(9.31) \\
47.96(11.45) \\
24.41(3.78) \\
54.85(7.96) \\
52.67(8.51) \\
51.70(8.82) \\
59.33(12.74) \\
57.78(12.98) \\
47.33(11.28) \\
57.11(10.39) \\
57.04(9.98) \\
55.19(9.32) \\
18.40(1.93) \\
19.75(1.74) \\
57.67(8.10) \\
55.74(8.07) \\
52.22(11.19) \\
53.81(8.32) \\
58.07(10.16) \\
52.63(9.03) \\
47.89(6.38) \\
55.85(8.43) \\
52.67(10.38) \\
50.41(8.54) \\
50.22(5.92) \\
50.59(8.11) \\
47.19(9.45) \\
48.15(8.71) \\
52.78(10.69) \\
46.78(8.50) \\
47.48(9.89) \\
49.67(10.42) \\
47.63(8.80) \\
45.74(10.08) \\
920.37(115.09) \\
92 \%(5 \%) \\
\end{gathered}
$$

0.2040

0.4860

0.0030

0.8330

0.2800

0.2610

0.0000

0.4090

0.0010

0.2710

0.3470

0.1160

0.0060

0.4000

0.8660

0.6250

0.5310

0.0680

0.8660

0.6520

0.2510

0.1540

0.0000

0.0010

0.0010

0.0170

0.0930

0.0030

0.0130

0.1160

0.0120

0.0000

0.0130

0.0050

0.0040

0.1200

0.0180

0.1560

0.0030

0.1420

0.0840

0.3650

0.0170

0.0640

0.0180

0.5780

0.2270

0.0120 


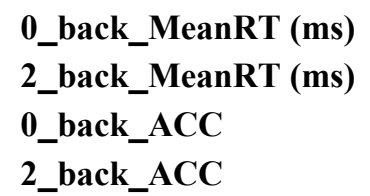

All continuous variables are shown as mean (std). All variables are described in Supplementary Table 5. $P$-values reflect group differences from either a $t$-test or a chi-squared test as appropriate.

\section{Supplementary Table 5. Description of the 59 behavioral variables.}

\begin{tabular}{|c|c|}
\hline Variable Name & Variable Description \\
\hline Sex & Male and Female \\
\hline Age & Age in years \\
\hline BMI & Current Body Mass Index \\
\hline $\mathrm{HbA1c}$ & Hemoglobin A1C \\
\hline EAG & Estimated average glucose measured $(\mathrm{mg} / \mathrm{dl})$ \\
\hline Estradiol & Mean of two results of the same sample $(\mathrm{pg} / \mathrm{ml})$ \\
\hline Testosterone & Mean of two results of the same sample $(\mathrm{pg} / \mathrm{ml})$ \\
\hline High Blood Pressure & Personal history of high blood pressure. Yes or No \\
\hline Number of Alcoholic Drinks & $\begin{array}{l}\text { Average number of alcoholic drinks consumed/month. Computed to Likert scale } \\
\text { score }(0-8 \text {, with } 0 \text { : no drinks, and } 8 \text { : daily drinks })\end{array}$ \\
\hline Physical Activity & $\begin{array}{l}\text { Composite score reflecting the degree of intensity and frequency of exercise } \\
\text { activity. Computed scale score ranging ( } 0-6 \text {, with } 0 \text { : no activity, } 6 \text { : daily } \\
\text { exercise with high intensity). }\end{array}$ \\
\hline Weeknight Sleep & Average hours of sleep on a weeknight \\
\hline Weekend Night Sleep & Average hours of sleep on a weekend night \\
\hline MMSE_total & Total score for the Mini-Mental Status Exam \\
\hline Block_design & $\begin{array}{l}\text { Raw score for the block design section on the WASI-II. Participants are asked to } \\
\text { re-create the block pattern they are shown as quickly as they are able. }\end{array}$ \\
\hline Vocabulary & $\begin{array}{l}\text { Raw score for the vocabulary section on the WASI-II. Participants are asked to } \\
\text { define the meaning of words they are given. }\end{array}$ \\
\hline Matrix_reasoning & $\begin{array}{l}\text { Raw score for the matrix reasoning section on the WASI-II. Participants are } \\
\text { asked to identifying the design to complete the pattern shown. }\end{array}$ \\
\hline Similarities & $\begin{array}{l}\text { Raw score for the similarities section on the WASI-II. Participants are asked to } \\
\text { identify the similarity between two words. }\end{array}$ \\
\hline FSIQ & $\begin{array}{l}\text { Full Scale IQ score taken from sum of T-Scores on the WASI-II combining the } \\
\text { block design, vocabulary, matrix reasoning, and similarities sections }\end{array}$ \\
\hline Total_FAS & $\begin{array}{l}\text { Verbal Fluency. Sum of total number of words participant verbalizes starting } \\
\text { with a F, A and S in one minute each. }\end{array}$ \\
\hline Animal_name & $\begin{array}{l}\text { Semantic Fluency. Sum of total number of animals participant verbalizes in one } \\
\text { minute. }\end{array}$ \\
\hline cog_orr_t & $\begin{array}{l}\text { Oral reading recognition T-Score. Participant is asked to read and pronounce } \\
\text { letters and words as accurately as possible. Completed via NIH Toolbox. }\end{array}$ \\
\hline $\operatorname{cog} \_p v \_t$ & $\begin{array}{l}\text { Picture Vocabulary T-Score. Participant selects the picture (out of four pictures } \\
\text { presented) that most closely matches the meaning of the word. Completed via } \\
\text { NIH Toolbox. }\end{array}$ \\
\hline cog_lswm_t & $\begin{array}{l}\text { List Sorting Working Memory T-Score. Participant is asked to verbally repeat } \\
\text { back objects that were shown with an auditory explanation, but re-order them by } \\
\text { size from smallest to largest. The list of objects continually becomes longer. } \\
\text { Completed via NIH Toolbox. }\end{array}$ \\
\hline cog_pcps_t & $\begin{array}{l}\text { Pattern Comparison Processing Speed T-Score. Participant is asked to } \\
\text { indicate whether two pictures are matching or not. Items are simple so as to } \\
\text { purely measure processing speed. They are asked to respond as quickly and } \\
\text { accurately as they are able to. Completed via NIH Toolbox. }\end{array}$ \\
\hline
\end{tabular}


cog_psm_t

cog_fica_t

cog_dccs_t

$\operatorname{cog}$ fc $t$

cog_cc_t

pegboard_RH

pegboard LH

emo_ahff_t

emo_aaff t

emo_apaff_t

emo_phff_t

emo_lff_t

emo_prff_t

emo_secat_t

emo_psff_t

emo_faff_t

emo_fsaff_t

emo_glsff_t

emo_dslsff raw

emo_mpff_t

emo_paff_t

emo_sff_t

emo_aff_t

emo_esff_t

emo_fff_t

emo_isff_t

emo_sss_t
Picture Sorting Memory T-Score. Participant views brief action activities presented as pictures with auditory explanation on a screen and attempt to place the pictures back in the same order they were shown on the screen. Completed via NIH Toolbox

Flanker Inhibitory Control and Attention T-Score. Participant must indicate which direction the middle arrow is pointing in a row of five arrows as quickly and accurately as they are able.

Dimensional Card Change Sort T-Score. Respondents are asked to indicate either the matching color or shape of a target picture based upon the prompt they were given as quickly and accurately as they are able. Completed via NIH Toolbox.

The fluid (intelligence) composite $\mathrm{T}$ score for the NIH Toolbox including the dimensional card change sort, flanker inhibitory, picture sequence memory, list sorting, and pattern comparison measures.

The crystalized (intelligence) composite score for the NIH Toolbox including picture vocabulary and oral reading recognition.

Participants are asked to place pegs, with their right hand, in holes one at a time as quickly as they are able and then take them out of the holes one at a time as they are able. Total time (sec). Completed with a physical peg board and the NIH Toolbox.

Participants are asked to place pegs, with their left hand, in holes one at a time as quickly as they are able and then take them out of the holes one at a time as they are able. Total time (sec). Completed with a physical peg board and the NIH Toolbox.

Anger and hostility questions. Computed T-Score. Completed via NIH Toolbox.

Anger with affect questions. Computed T-Score. Completed via NIH Toolbox. Anger with physical aggression questions. Computed T-Score. Completed via NIH Toolbox.

Perceived hostility questions. Computed T-Score. Completed via NIH Toolbox.

Loneliness questions. Computed T-Score. Completed via NIH Toolbox.

Perceived rejection questions. Computed T-Score. Completed via NIH Toolbox.

Self-efficacy questions. Computed T-Score. Completed via NIH Toolbox.

Perceived stress questions. Computed T-Score. Completed via NIH Toolbox.

Fear with affect questions. Computed T-Score. Completed via NIH Toolbox.

Fear with somatic arousal questions. Computed T-Score. Completed via NIH Toolbox.

General life satisfaction questions. Computed T-Score. Completed via NIH Toolbox.

Domain-specific life satisfaction questions. Computed raw score. Completed via NIH Toolbox.

Meaning and purpose questions. Computed T-Score. Completed via NIH Toolbox.

Positive affect questions. Computed T-Score. Completed via NIH Toolbox.

Sadness questions. Computed T-Score. Completed via NIH Toolbox.

Apathy questions. Computed T-Score. Completed via NIH Toolbox.

Emotional support questions. Computed T-Score. Completed via NIH

Toolbox.

Friendship questions. Computed T-Score. Completed via NIH Toolbox. Instrumental support questions. Computed T-Score. Completed via NIH Toolbox.

Social satisfaction summary T-Score. Completed via NIH Toolbox. 


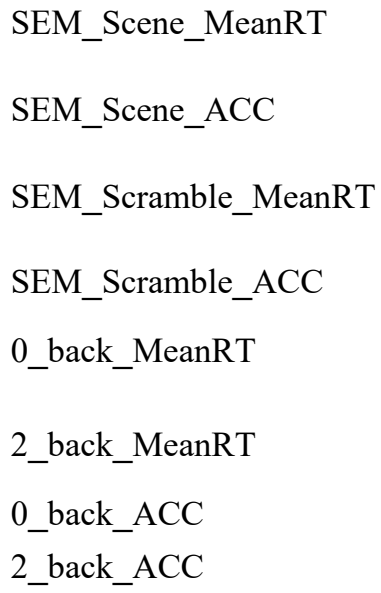

During the SEM fMRI task, mean reaction time (in $\mathrm{ms}$ ) for accurate responses only during the scene condition (participant is asked to determine if the picture is an indoor or outdoor setting).

During the SEM fMRI task, total accuracy (in \%) for the scene condition.

During the SEM fMRI task, mean reaction time (in $\mathrm{ms}$ ) for accurate responses only during the control condition (participant is asked to determine if the scrambled picture has 2 identical halves).

During the SEM fMRI task, total accuracy (in \%) for the control condition.

During the n-back fMRI task, mean reaction time (in $\mathrm{ms}$ ) for accurate responses only during the 0 -back condition.

During the $n$-back fMRI task, mean reaction time (in $\mathrm{ms}$ ) for accurate responses only during the 2-back condition.

During the n-back fMRI task, total accuracy (in \%) for the 0-back condition.

During the n-back fMRI task, total accuracy (in \%) for the 2-back condition.

Supplementary Table 6. Activation peak for the N-Back task.

\begin{tabular}{lcccc}
\hline Region & T & $\mathbf{x}$ & $\mathbf{y}$ & $\mathbf{z}$ \\
\hline Left Inferior Parietal Cortex & 6.81 & -38 & -50 & 46 \\
Right Inferior Parietal Cortex & 6.78 & 38 & -46 & 46 \\
Left Middle Frontal Cortex & 6.26 & -22 & 6 & 54 \\
Left Inferior Parietal Cortex & 6.18 & -50 & -44 & 46 \\
Right Middle Frontal Cortex & 7.84 & 28 & 8 & 52 \\
Left SMA & 6.42 & -6 & 12 & 50 \\
Right Cerebellum & 7.58 & 30 & -58 & -34 \\
Right Precuneus & 7.30 & 10 & -60 & 56 \\
Left Cerebellum & 7.06 & -42 & -62 & -36 \\
Left Middle Frontal Cortex & 5.63 & -30 & 44 & 16 \\
Right Thalamus & 5.22 & 14 & -8 & 10 \\
Left Insula & 4.92 & -32 & 16 & 2 \\
\hline
\end{tabular}

MNI Coordinates. Abbreviation: SMA: Supplementary Motor Area. Activation peaks are shown on Supplementary Figure 2B.

Supplementary Table 7. Activation peak for the scene encoding memory task.

\begin{tabular}{lcccc}
\hline Region & $\mathbf{T}$ & $\mathbf{x}$ & $\mathbf{y}$ & $\mathbf{z}$ \\
\hline Right Fusiform Gyrus & 18.01 & 26 & -46 & -10 \\
Left Fusiform Gyrus & 17.22 & -20 & -36 & -16 \\
Right Lingual Gyrus & 18.48 & 6 & -76 & -4 \\
Right Retrosplenial Cortex & 18.42 & 10 & -52 & 8 \\
Left Retrosplenial Cortex & 18.18 & -10 & -56 & 10 \\
Left Orbital MPFC & 13.36 & -6 & 20 & -14 \\
Left Middle Occipital Cortex & 12.70 & -46 & -72 & 26 \\
Right Middle Occipital Cortex & 11.76 & 48 & -76 & 2 \\
Right Middle Temporal Cortex & 9.73 & 56 & -2 & -22 \\
\hline
\end{tabular}

MNI Coordinates. Abbreviation: MPFC: Medial Prefrontal Cortex. 
Supplementary Table 8. Activation peak for the verb generation task.

\begin{tabular}{lcccc}
\hline Region & $\mathbf{T}$ & $\mathbf{x}$ & $\mathbf{y}$ & $\mathbf{z}$ \\
\hline Right Cerebellum & 18.25 & 30 & -60 & -30 \\
Left SMA & 16.02 & -8 & 4 & 64 \\
Left Inferior Frontal Gyrus & 15.27 & -48 & 12 & 6 \\
Left Cerebellum & 13.07 & -42 & -64 & -28 \\
Right anterior Insula & 11.94 & 42 & 16 & -4 \\
Left Inferior Parietal & 10.10 & -30 & -62 & 42 \\
Left Hippocampus & 10.09 & -32 & -18 & -10 \\
Right Putamen & 10.00 & 20 & 2 & 10 \\
\hline
\end{tabular}

MNI Coordinates. Abbreviation: SMA: Supplementary Motor Area. 